ARTICLE

Received 1 Sep 2015 | Accepted 25 Dec 2015 | Published 4 Feb 2016

DOI: $10.1038 /$ ncomms10549

OPEN

\title{
Chromatin remodeller SMARCA4 recruits topoisomerase 1 and suppresses transcription-associated genomic instability
}

Afzal Husain ${ }^{1}$, Nasim A. Begum ${ }^{1}$, Takako Taniguchi ${ }^{2}$, Hisaaki Taniguchi ${ }^{2}$, Maki Kobayashi ${ }^{1}$ \& Tasuku Honjo ${ }^{1}$

Topoisomerase 1, an enzyme that relieves superhelical tension, is implicated in transcriptionassociated mutagenesis and genome instability-associated with neurodegenerative diseases as well as activation-induced cytidine deaminase. From proteomic analysis of TOP1-associated proteins, we identify SMARCA4, an ATP-dependent chromatin remodeller; FACT, a histone chaperone; and H3K4me3, a transcriptionally active chromatin marker. Here we show that SMARCA4 knockdown in a B-cell line decreases TOP1 recruitment to chromatin, and leads to increases in $\mathrm{gh} / \mathrm{c}-\mathrm{Myc}$ chromosomal translocations, variable and switch region mutations and negative superhelicity, all of which are also observed in response to TOP1 knockdown. In contrast, FACT knockdown inhibits association of TOP1 with H3K4me3, and severely reduces DNA cleavage and $\mathrm{lgh} / \mathrm{c}-\mathrm{Myc}$ translocations, without significant effect on TOP1 recruitment to chromatin. We thus propose that SMARCA4 is involved in the TOP1 recruitment to general chromatin, whereas FACT is required for TOP1 binding to H3K4me3 at non-B DNA containing chromatin for the site-specific cleavage.

\footnotetext{
${ }^{1}$ Department of Immunology and Genomic Medicine, Graduate School of Medicine, Kyoto University, Yoshida-Konoe cho, Sakyo-ku, Kyoto 606-8501, Japan ${ }^{2}$ Division of Disease Proteomics, Institute for Enzyme Research, University of Tokushima, Tokushima 770-8503, Japan. Correspondence and requests for materials should be addressed to T.H. (email: honjo@mfour.med.kyoto-u.ac.jp).
} 
T opoisomerase 1 (TOP1) is an enzyme that relieves the superhelical tension generated when the transcription machinery travels along DNA. Normally, TOP1 nicks DNA, forms a transient covalent bond between its tyrosine residue and the $3^{\prime}$ phosphate of the nicked DNA, rotates DNA around the helix, and then re-ligates the cleaved ends to release negative or positive supercoils that accumulate behind or in front, respectively, of elongating RNA polymerase II $(\mathrm{Pol} \mathrm{II})^{1,2}$. High levels of transcription can lead to the accumulation of negative supercoils behind RNA pol II and facilitate the formation of nonB DNA structures, particularly at repeat-containing sequences. These changes in DNA structure are likely due to insufficient levels of TOP1. In fact, TOP1 reduction by small interfering RNA (siRNA) induces high levels of genomic instability ${ }^{2-4}$. The accumulated non-B DNA structures may promote genomic instability by posing steric hindrance in the rotation or re-ligation of cleaved ends following DNA nicking by TOP1, resulting in irreversible DNA cleavage ${ }^{2-10}$.

TOP1 was recently shown to be responsible for 2-5-bp deletions that occur during transcription-associated mutagenesis, and the triplet-repeat instability associated with triplet diseases such as Huntington's disease ${ }^{11-14}$. In addition, defects in DNA damage response factors involved in the processing of TOP1-DNA lesions, such as ataxia telangiectasia mutated, TDP1 (tyrosyl-DNA phosphodiesterase 1), Aprataxin and PNKP (polynucleotide kinase phosphatase), are implicated in neurodegenerative genome instability syndromes such as ataxia telangiectasia, spinocerebellar ataxia with axonal neuropathy 1 , ataxia with oculomotor apraxia type 1 , and microcephaly with early-onset, intractable seizures and developmental delay, respectively ${ }^{15-18}$. It is important to stress that these genome instabilities in the nerve cells do not depend on replication, but depend on transcription in which TOP1 plays a critical role.

Another enzyme associated with genomic stress is activationinduced cytidine deaminase (AID), which is normally responsible for the DNA cleavage in the switch $(\mathrm{S})$ and variable $(\mathrm{V})$ regions of the immunoglobulin (Ig) locus, which initiates class switch recombination (CSR) and somatic hypermutation (SHM), respectively ${ }^{19-21}$. Aberrant expression of AID induces a high frequency of mutations and chromosomal translocations in B as well as non- $B$ cells that eventually leads to increased tumour formation ${ }^{22}$. It has also been shown that the activation of AID reduces TOP1 protein expression ${ }^{23}$, while the artificial reduction of TOP1 by siRNA augments AID-induced DNA cleavage, SHM and $\mathrm{CSR}^{23,24}$. Furthermore, the reduced expression of TOP1 in Top1 heterozygous knockout mice leads to a dramatic increase in the frequency of SHM in Peyer's patch B cells ${ }^{24}$. These findings indicate that reduction in the TOP1 protein level enhances the DNA cleavage that augments SHM or CSR. However, trapping the TOP1-DNA intermediates by camptothecin inhibits both CSR and SHM, suggesting that the appropriate processing of TOP1-DNA lesions is also required for SHM and CSR. Taken together, these findings suggest that AID-dependent chromosomal translocations may serve as a model to study TOP1-mediated genomic instability.

Preferred targets of TOP1-mediated genomic instability are highly transcribed and enriched in repetitive sequences ${ }^{1-13}$. Notably, the targeted loci of AID-induced DNA cleavage also share similar properties ${ }^{25-28}$. Ig gene targeting by AID has been proposed to depend not only on the cis marking by secondary DNA structures such as non-B DNAs, produced by excessive transcription at repeat-containing AID targets, but also on trans marking by a variety of chromatin factors, including histone $\mathrm{H} 3$ tri-methyl at Lys4 (H3K4me3) ${ }^{23,26,29}$. Consistent with this proposal, transcription elongation factors, such as FACT (a heterodimeric complex composed of SSRP1 and
SPT16/SUPT16H), SPT6/SUPT6H, SPT5/SUPT5H and H3K4me3methyltransferases, which are required for the maintenance of H3K4me3, are essential for AID-induced genetic alterations ${ }^{29-32}$. Furthermore, non-canonical AID targets such as MYC, MALAT1 and $S N H G 3$, identified in a genome-wide study, are highly transcribed, flanked by repetitive sequences, and enriched with H3K4me3 as well as FACT $25,26,32,33$.

There is evidence that a large number of chromatin-modifying proteins are also involved in the maintenance of genomic stability. Among these, proteins found in the SWI/SNF chromatin remodelling complexes, which are also referred to as BAF (BRG1-associated factor) complexes, are frequently mutated in human cancers, suggesting that the inactivation of these proteins may induce the genomic instability that contributes to tumorigenesis ${ }^{34,35}$. However, the mechanisms by which BAF complex inactivation cause genomic instability are not well understood. The BAF complexes are highly polymorphic assemblies containing ATPase SMARCA4 (also called BRG1), which utilizes ATP hydrolysis to modulate non-covalent chromatin structural changes by destabilizing, removing, sliding and restructuring nucleosomes ${ }^{36,37}$.

To understand the molecular mechanisms involved in TOP1-mediated genomic instability, we sought to identify proteins responsible for TOP1 recruitment to chromatin. We performed TOP1 co-immunoprecipitations (co-IPs) followed by protein identification by mass spectrometry (MS) to identify proteins that associate with TOP1. Among the co-precipitated proteins, we found that SMARCA4 is involved in the recruitment of TOP1 to chromatin. Some of the recruited TOP1 interacts with the FACT that accumulates at non-B DNA containing chromatin, and forms a complex with the chromatin mark H3K4me3. Subsequently, the TOP1 complex induces breaks at non-B DNA formed at the repeat containing DNA sequences. Our findings indicate that SMARCA4 and FACT are involved in the regulation of TOP1-dependent genomic instability associated with transcription.

\section{Results}

Proteomic analysis of TOP1-associated proteins. We carried out co-immunoprecipitation experiments using a transfectant of the TOP1-deficient mouse B-lymphocytic leukemia cell-line P388/CPT45, that stably expresses GFP-tagged human TOP1 (GFP-TOP1) and the estrogen-binding domain (ER)-AID (AIDER) fusion protein ${ }^{24}$. Immunoprecipitations (IPs) were performed using the GFP-Trap system, followed by protein identification with one-dimensional SDS-PAGE (SDS polyacrylamide gel electrophoresis) separation and mass spectrometry.

We used dithiobis-succinimidyl propionate (DSP), a cellpermeable and thiol-reversible chemical crosslinker, to identify labile but functionally relevant TOP1-interacting proteins. To generate a real-time snapshot of the TOP1-protein interactome in vivo, AID-activated P388/CPT45-GFP-TOP1 transfectants were treated with DSP $(0.6 \mathrm{mM})$ for $30 \mathrm{~min}$, followed by IP (DSP-IP) and MS-identification of the immunoprecipitated (IPed) proteins (Fig. 1a-d). Under these conditions, TOP1 was efficiently crosslinked to its interacting partners, and the IPed fraction contained only the crosslinked form of TOP1, which was thiol-reversible (Supplementary Fig. 1a,b). To overcome the shortcomings of DSP-IP, such as nonspecific protein trapping, we also performed IP without crosslinking (native-IP), although proteins with labile TOP1 interactions may be lost with this method (Fig. 1a-d; Supplementary Fig. 1c). We postulated that molecules commonly trapped by both methods may be a more reliable representation of the TOP1 interactome than molecules identified by only one method. 

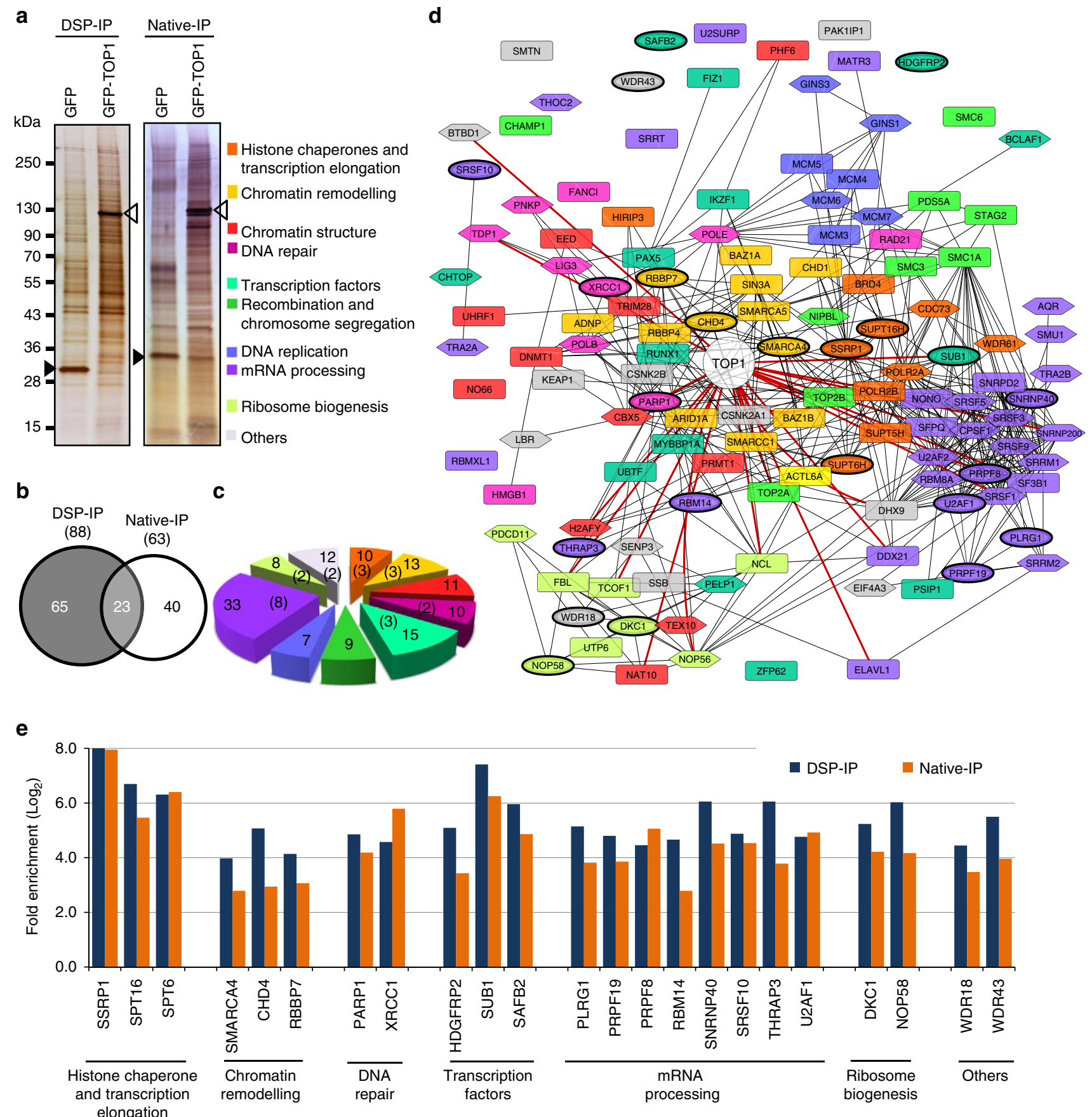

Figure 1 | Proteome-wide identification of TOP1-associated proteins. (a) Representative silver-stained gels of protein fractions obtained from GFP-TOP1 IPs performed under native (Native-IP) or DSP-cross-linked conditions (DSP-IP). Immunoprecipitations were performed with GFP-Trap_A using nuclear extracts prepared from $2 \times 10^{8}$ DSP treated or untreated P388/CPT45 cells expressing either GFP (P388/CPT45-GFP) or GFP-TOP1 (P388/CPT45-GFP-TOP1) as described in the 'Methods'. The IPed proteins were electrophoresed on SDS-PAGE gel (4-20\% acrylamide gel) and visualized with SilverQuest Silver Staining Kit (Invitrogen). Open and closed triangles represent the protein bands corresponding to GFP-TOP1 or GFP alone, respectively. For MS analysis, IPed proteins were processed as described in the 'Methods'. (b) Venn diagram representing LC-MS/MS identified TOP1-associated proteins obtained by DSP- or native-IP. The number of proteins identified by each method is shown in parentheses. The complete list of MS identified proteins is shown in Supplementary Tables 1 and 2. (c) Functional classification of TOP1-associated proteins. The number of common proteins identified by both methods in the various groups is shown in parentheses. (d) Physical and functional interactions among the TOP1-associated proteins based on information obtained from the STRING 9.1 database (http://string-db.org). Proteins identified by both methods are shown in ellipses, whereas those identified by either DSP-IP or native-IP are shown in rectangles or hexagons, respectively. Red coloured edges represent known TOP1 associations identified in the STRING database. Proteins that are not connected with rest of the network showed no evidence of association with TOP1 or the other network proteins in the STRING database. The colour of each node represents the functional class shown on the left. (e) Fold enrichment of the common proteins identified by GFP-TOP1 IP compared with their levels in GFP IPs from control cells. The fold enrichments of all the proteins identified by each method are shown in Supplementary Tables 1 and 2. DSP, dithiobis-succinimidyl propionate. 
The MS analysis of proteins obtained by DSP- and native-IP from GFP-TOP1-expressing cells led to the identifications of 88 and 63 proteins, respectively (Supplementary Tables 1 and 2). Among the co-IPed proteins, 65 proteins were identified by DSP-IP alone, 40 proteins were identified by native-IP alone, and 23 proteins were identified by both methods (Fig. 1b). Theoretically, all the proteins that were identified by native-IP should be picked up by DSP-IP due to their further stabilization by crosslinking. However, despite stabilization of the labile interactions, crosslinking negatively affects the extraction of many protein complexes likely due to their crosslinking with other non-soluble proteins such as those in the nuclear matrix, and thus limits their successful IP and identification by MS. The functional classification of the 128 unique TOP1-associated proteins suggests that TOP1 may be a central component of a large protein interaction network associated with transcription, DNA repair and other chromatin-related functions (Fig. 1c).

To further elucidate the functional relationships among the TOP1-associated proteins, we mapped them using the STRING database, a knowledge base of protein associations, and visualized the interactions by Cytoscape 3.2 (refs 38,39). As shown in Fig. 1d, $113(88 \%)$ of these proteins were connected by a functional network, suggesting that the TOP1-associated proteins were functionally interrelated. Notably, only 28 of the 113 proteins in this network were previously known to associate with TOP1; these included SRSF1, NCL, ELAVL1, FBL, NONO, SFPQ, TCOF1, PARP1 and BTBD1. One of the major pathways implicated in the repair of DNA damage induced by TOP1-DNA adducts requires proteolysis of TOP1 by proteasome, excision by TDP1, followed by repair that requires the action of XRCC1 complex proteins such as PNKP, PARP1, LIGIII and POLB. Interestingly, all of these were identified in our proteomic studies (Supplementary Tables 1 and 2$)^{40}$. These results indicate that the experimental strategies described here identified both known and novel TOP1-associated proteins.

TOP1-associated proteins are required for efficient CSR. We focused on the proteins identified by both methods, which included histone chaperones and transcription elongation factors (SSRP1, SPT16, SPT6), chromatin remodelling complex proteins (SMARCA4, CHD4 and RBBP7), DNA repair proteins (PARP1 and XRCC1) and proteins involved in splicing and ribosome biogenesis (Fig. 1e). In addition to SMARCA4, other components of the BAF complex such as SMARCC1, ARID1A and ACTL6A were also identified by DSP-IP (Supplementary Table 1). To investigate the functional relevance of these proteins in TOP1 recruitment, we performed siRNA-mediated knockdown (KD) of these proteins in $\mathrm{CH} 12 \mathrm{~F} 3-2 \mathrm{~A}$ cells, and assessed the effects on IgM to IgA switching in response to stimulation with CD40L, IL4, and TGF $\beta$ (CIT) (Fig. 2a). We were particularly interested in the proteins involved in chromatin modification because of their likely involvement in recruiting TOP1 to chromatin. The KD of the transcription elongation-associated histone chaperones, SPT6 and FACT (SSRP1 and SPT16) strongly reduced CSR as previously shown $29,30,41$. The $\mathrm{KD}$ of the chromatin remodelling proteins SMARCA4, CHD4 and RBBP7 led to 70\%, 50\% and $30 \%$ reductions in CSR, respectively (Fig. 2a; Supplementary Fig. $2 \mathrm{a}-\mathrm{c}$ ). Thus, we concentrated on the role of SMARCA4 and FACT in TOP1 recruitment, as SPT6 is known to directly regulate AID expression.

We confirmed that both SMARCA4 and FACT (SSRP1 and SPT16 subunits) were specifically IPed from GFP-TOP1expressing cells, but not from control cells expressing GFP alone (Fig. 2b; Supplementary Fig. 1d-f). In addition, we confirmed the interaction of TOP1 with other transcription-associated proteins, such as RNA pol II and SPT5, a subunit of the DSIF (DRB sensitivity inducing factor) complex, which were also found in the proteomic analysis, albeit with lower enrichment. These results not only validated the proteomic data, but also suggested that recruitment of TOP1 may be transcription-dependent. Consistent with this notion, treating $\mathrm{CH} 12 \mathrm{~F} 3-2 \mathrm{~A}$ cells with the transcription inhibitors DRB (5,6-Dichloro-1- $\beta$-D-ribofuranosylbenzimidazole, $25 \mu \mathrm{M}$ for $24 \mathrm{~h}$ ) or ActD (actinomycin D, $2 \mu \mathrm{M}$ for $4 \mathrm{~h}$ ) dramatically decreased the TOP1-ChIP (chromatin immunoprecipitation) signal at the Igh locus (Supplementary Fig. 3). To gain insight into the role of epigenetic marking on TOP1 recruitment, the TOP1-co-IPed proteins were analysed for the presence of several histone post-translational modifications (PTMs). We found that TOP1-associated chromatin was highly enriched in $\mathrm{H} 3 \mathrm{~K} 4 \mathrm{me} 3$ as compared with other tested histonePTMs (H3-acetyl, H4-acetyl, H3S10P, H3S28P, H3K27me3 and H3K9me3; Fig. 2c). These data suggest that TOP1 may preferentially bind to $\mathrm{H} 3 \mathrm{~K} 4 \mathrm{me} 3$, and be likely enriched in the $\mathrm{H} 3 \mathrm{~K} 4 \mathrm{me} 3$-modified genomic regions.

SMARCA4 depletion augments AID-induced genomic instability. The KD of SMARCA4 in CH12F3-2A cells with three individual siRNAs or their pool inhibited IgA switching without causing significant cell death (Supplementary Figs $2 a-c$ and $4 a-d$ ), and reduced the expression of $\alpha$-germline transcripts (GLTs) (Supplementary Fig. 2d). Drastic reduction of SMARCA4 by siRNA had a very limited effect on cell proliferation, suggesting that CSR inhibition upon SMARCA4 KD is largely due to the inhibition of $\alpha$ GLT expression (Supplementary Fig. 2d,e). To further confirm this possibility, we performed the CSR assay using an artificial switch substrate in AIDER expressing NIH3T3 cells ${ }^{42}$. SMARCA4 KD in these cells reduced neither CSR nor the expression of $S \mu$ or $S \alpha$ transcripts (Pre-Tr1 and Pre-Tr2) from the artificial promoters (Supplementary Fig. 2f-h). The data further confirmed that CSR defect upon SMARCA4 KD in CH12F32-A cells is due to the inhibition of $\alpha$ GLT expression.

We next analysed the role of SMARCA4 in SHM. To determine the effect of SMARCA4 KD on V region SHM, we used AID-knockout BL2 cells (human Burkitt's lymphoma line) expressing AIDER (BL2-AID ${ }^{-l}$ - AIDER) ${ }^{43,44}$. After transfection with siRNA-targeting human SMARCA4, the cells were treated with 4-hydroxytamoxifen (OHT) from days 1 to 4 to activate $\mathrm{AID}$, and the 426-bp rearranged $\mathrm{V}_{\mathrm{H}} 4-39-\mathrm{J}_{\mathrm{H}} 5$ region was sequenced (Fig. 3a). Transfection of SMARCA4 siRNA sufficiently suppressed SMARCA4 protein expression, as compared with the effect of control non-targeting siRNA with a similar GC content (Fig. 3b). The KD of SMARCA4 increased the SHM frequency in the $\mathrm{V}(\mathrm{D}) \mathrm{J}$ region induced by AIDER activation (Fig. 3c; Supplementary Tables 3 and 4).

As the enhancement of SHM by SMARCA4 KD is reminiscent of that induced by TOP1 depletion, we speculated that SMARCA4 depletion in BL2 cells may affect TOP1 function, which led to enhanced DNA cleavage and SHM. To test this possibility, we analysed the effect of SMARCA4 KD on SHM in the $\mathrm{S} \mu$ region of the TOP1-deficient P388/CPT45 cells expressing either GFP (P388/CPT45-GFP) or GFP-TOP1 (P388/CPT45GFP-TOP1; Fig. 3d-g; Supplementary Tables 5 and 6). Introducing SMARCA4 siRNA into these cells efficiently reduced the SMARCA4 protein expression (Fig. 3f). As reported previously ${ }^{24}$, expression of GFP-TOP1 in the TOP1-deficient P388/CPT45 cells resulted in a clear reduction in SHM frequency (Fig. 3g). Notably, SMARCA4 depletion rescued the decrease in SHM frequency in the P388/CPT45-GFP-TOP1 cells, suggesting that SMARCA4 reduction counteracts TOP1 overexpression. Interestingly, SHM augmentation by SMARCA4 KD was not observed in TOP1-deficient P388/CPT45-GFP cells, confirming that SMARCA4 depletion and TOP1 deficiency are functionally 
a

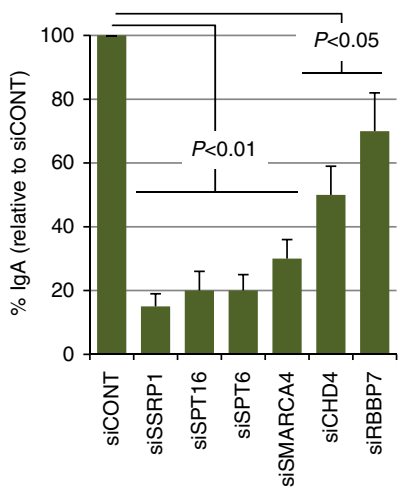

b

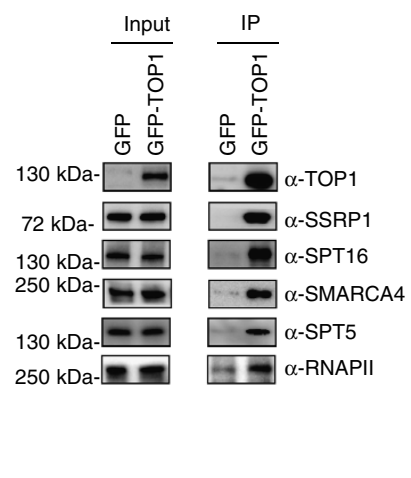

C

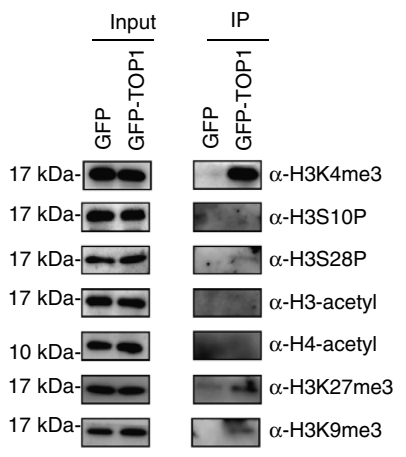

Figure 2 | TOP1-associated proteins are required for efficient CSR. (a) Effect of siRNA-mediated KD of histone chaperones and chromatin remodellers on IgM to IgA switching in $\mathrm{CH} 12 \mathrm{~F} 3-2 \mathrm{~A}$ cells. After electroporation of the indicated siRNAs, cells were cultured for $24 \mathrm{~h}$, and then stimulated by $\mathrm{CIT}$ for another $24 \mathrm{~h}$ before FACS analysis. Data are presented as \% IgA switching relative to the IgA switching in cells transfected with control siRNA (siCONT) with similar GC content. The data represents the mean of the three independent experiments with standard deviations. Statistical significance as evaluated by Student's $t$-test is shown. (b) Confirmation of association of TOP1 with FACT, SMARCA4 and other transcription-associated proteins. The nuclei from $2 \times 10^{8}$ P388/CPT45 transfectants expressing either GFP (P388/CPT45-GFP) or GFP-TOP1 (P388/CPT45-GFP-TOP1) were IPed with GFP-Trap_A as described for native-IP in the 'Methods', and 5-10\% of the IPed proteins were electrophoresed on 4-20\% SDS-PAGE gradient gel, followed by western blot with indicated antibodies. (c) Association of TOP1 with H3K4me3 and other histone PTMs. Immunoprecipitated proteins obtained in $\mathbf{b}$ were immunoblotted to test for the presence of the indicated histone-PTMs using their specific antibodies. The position of molecular weight markers is shown on the left of each western blot image.

related. Since increased mutations may represent increments in the double-stranded breaks (DSBs), we analysed the effect of SMARCA4 depletion on the $\mathrm{H} 2 \mathrm{AX}$ phosphorylation. Indeed, depletion of SMARCA4 in $\mathrm{CH} 12 \mathrm{~F} 3-2 \mathrm{~A}$ cells resulted in the increased formation of AID-induced $\gamma \mathrm{H} 2 \mathrm{AX}$ foci in the $\mathrm{S} \mu$ region (Fig. 3h).

As TOP1 reduction is known to cause genomic instability, we assessed the effect of SMARCA4 KD on the chromosomal translocations involving the $c-M y c$ oncogene and Igh locus, a hallmark of various B-cell malignancies. The products of longrange PCR amplification of the translocated genomic DNA using Igh- and $c-M y c$-specific primers were detected by Southern blotting with $M y c$ probes (Fig. 4a). Depletion of either SMARCA4 or TOP1 with siRNA in CH12F3-2A cells led to dramatically increased translocation frequencies (Fig. 4b-d); however, the frequency of translocations upon TOP1 KD was higher than that observed upon SMARCA4 KD. This conclusion is further confirmed by the KD of SMARCA4 with the pool of three SMARCA4 siRNAs (Supplementary Fig. 4f). In addition, although SMARCA4 KD augmented the chromosomal translocation frequency in TOP1-expressing cells, it had no effect on chromosomal translocation in CH12F3-2A cells expressing microRNA directed against Top1 mRNA (Fig. 4e-g). To exclude the possibility that absence of the chromosomal translocation increase by SMARCA4 KD in TOP1-depleted cells is not due to saturation of the assay sensitivity, we performed the translocation assay using lesser amount of genomic DNA (300 ng instead of $750 \mathrm{ng}$ per PCR reaction). Even under this unsaturated assay conditions, we failed to see additive increments in the $I g h / c-M y c$ translocations (Supplementary Fig. 5). These results confirmed that the effect of SMARCA4 KD on chromosomal translocations was dependent on the presence of TOP1. In addition, we also showed that complementation of siRNA-mediated KD of SMARCA4 by the co-transfection of plasmid expressing siRNA-resistant WT-SMARCA4 abolished the increments in $I g h / c-M y c$ translocations (Supplementary Fig. 6).

FACT is essential for AID-induced genomic instability. We next investigated the role of FACT, another prominent component of the TOP1 complex, and found that FACT depletion in $\mathrm{CH} 12 \mathrm{~F} 3-2 \mathrm{~A}$ cells caused a dramatic reduction in the $I g h / c-M y c$ chromosomal translocations induced by AID expression (Fig. 5a-c,e,g). The results suggest that unlike TOP1 and SMARCA4, FACT facilitates AID-induced genomic instability. To further confirm this possibility, we assayed the AID-induced $I g h / c-M y c$ chromosomal translocations upon simultaneous KD of TOP1 and FACT in CH12F3-2A cells. Surprisingly, FACT KD abolished the TOP1 depletion-mediated augmentation of $I g h / c-M y c$ chromosomal translocations (compare Fig. 4c versus Fig. 5d,e). When we measured formation of DSBs using ligation-mediated PCR, FACT depletion severely reduced the formation of AID-induced DSBs, which is consistent with our previous studies ${ }^{29}$ (Fig. 5f). These data suggest that FACT expression is essential for DNA cleavage at $I g$ and $M y c$ loci, even in TOP1-deficient cells. Thus, FACT and SMARCA4 play distinct roles in AID-induced genomic instability.

SMARCA4 depletion reduces TOP1 recruitment to chromatin. Since TOP1 is required for augmentation of SHM and chromosomal translocations by SMARCA4 depletion, we speculated that SMARCA4 is involved in TOP1 deposition at the Igh locus, and thus we performed TOP1-ChIP using CH12F3-2A cells following transfection with SMARCA4 siRNA. Indeed, SMARCA4 depletion reduced deposition of TOP1 in the $\mathrm{S} \mu$ region, confirming that TOP1 association with SMARCA4 is functionally relevant and required for efficient TOP1 deposition at the Igh locus (Fig. 6a). The reduction in the recruitment of TOP1 upon SMARCA4 KD was further confirmed by TOP1-ChIP analysis upon SMARCA4 KD by the pool of three SMARCA4 siRNAs (Supplementary Fig. 4e). The reduction in association of TOP1 with chromatin causes the accumulation of negative supercoils behind elongating RNA Pol II, resulting in the formation of non-B DNA structures as well as single-stranded patches of $\mathrm{DNA}^{4,9,10,23,45}$. To evaluate the relative frequencies of negative superhelicity, we used biotin-trimethylpsoralen (bTMP), which preferentially intercalates into under-wound DNA, followed by ultraviolet-crosslinking, streptavidin-based IP of the crosslinked DNA, and quantification of the IPed DNA by quantitative PCR 
a
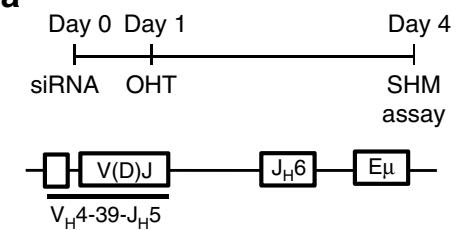

b

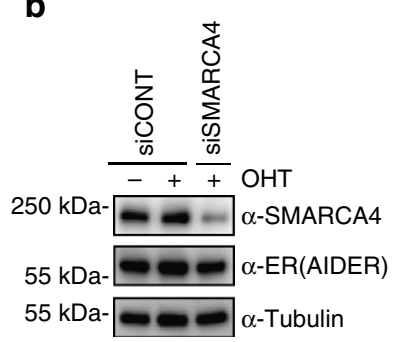

C

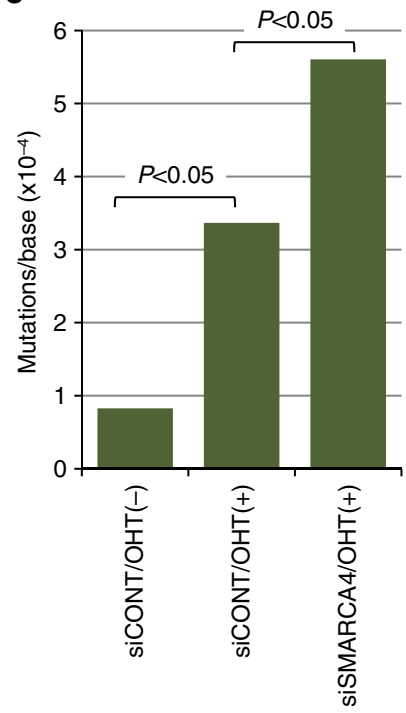

d

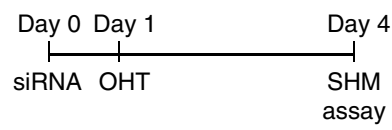

e

GFP-

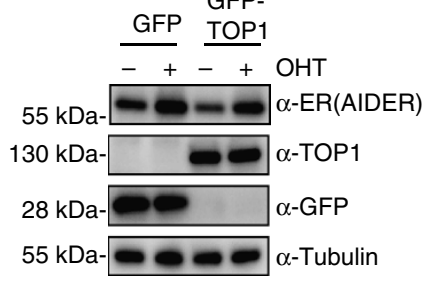

$\mathbf{f}$

GFP-

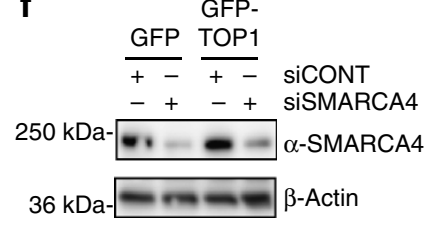

h
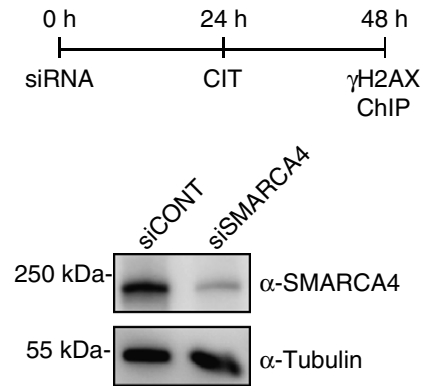
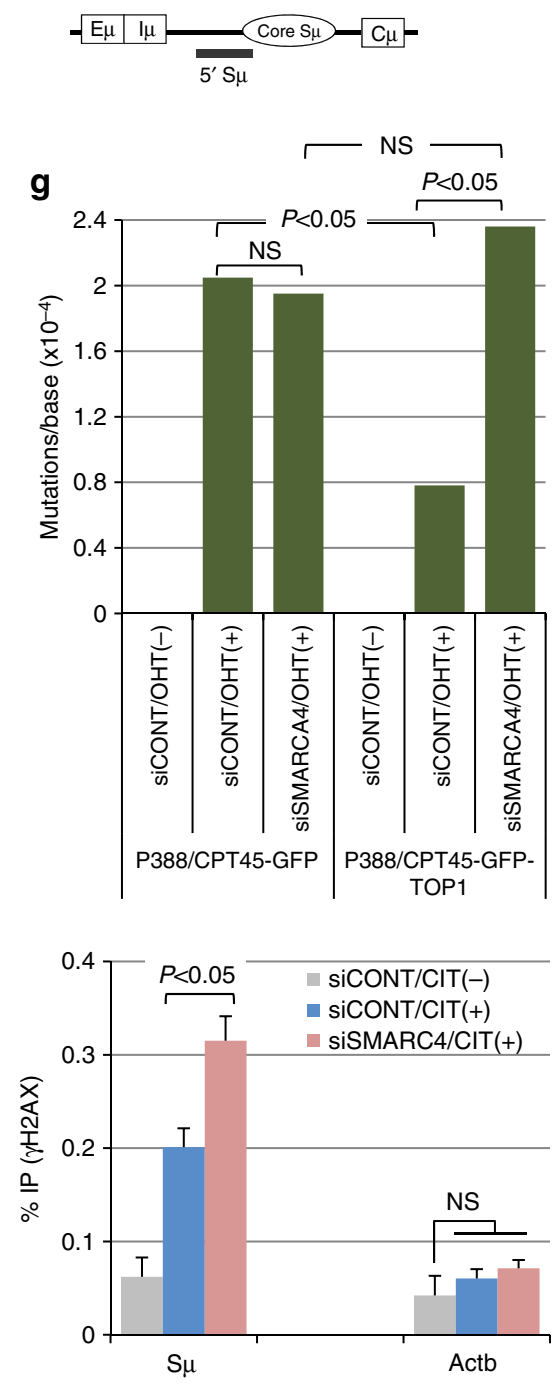

Figure 3 | SMARCA4 depletion augments SHM in a TOP1-dependent manner. (a) Top: experimental design for SHM analysis of the $\mathrm{V}$ region in BL2-AID ${ }^{-/-A I D E R}$ cells. Bottom: schematic representation of recombined V(D)J region of BL2-AID - /-AIDER cells. The region analysed for SHM is underlined. (b) Confirmation of siRNA-mediated KD of SMARCA4 in BL2-AID ${ }^{-/-}$AIDER cells. Expression of AIDER is also shown. Tubulin is used as loading control. (c) Mutation frequency in the $\mathrm{V}$ region after 3 days of AIDER activation by OHT treatment. Details of the mutation analysis are shown in Supplementary Tables 3 and 4. (d) Left: schematic illustration of the experimental design for SHM analysis of the S $\mu$ region in P388/CPT45 cells. Right: schematic representation of $\mathrm{S} \mu$ region of P388/CPT45 cells. The $5^{\prime} \mathrm{S} \mu$ region analysed for SHM is underlined. (e) Expression of GFP or GFP-TOP1, and AIDER in P388/CPT45 cells expressing either GFP (P388/CPT45-GFP) or GFP-TOP1 (P388/CPT45-GFP-TOP1) is analysed by western blotting with indicated antibodies. (f) Confirmation of siRNA-mediated KD of SMARCA4 in P388/CPT45 cells by western blotting with indicated antibodies. Expression of $\beta$-actin is shown as loading control. (g) Mutation frequency in the $S \mu$ region after 3 days of AIDER activation by OHT. Details of the mutation analysis are shown in Supplementary Tables 5 and 6. (h) Left: Schematic representation of the $\gamma \mathrm{H} 2 \mathrm{AX}$-ChIP assay using CH12F3-2A cells (top), and confirmation of SMARCA4 KD by western blot with anti-SMARCA4 antibody. Expression of tubulin is shown as a loading control. Right: $\gamma \mathrm{H} 2 \mathrm{AX}-\mathrm{ChIP}$ signal in S $\mu$ region is represented as the fraction of immunoprecipitated DNA (\%IP) normalized to the total amount of DNA used for immunoprecipitation. $\gamma \mathrm{H} 2 \mathrm{AX}-\mathrm{ChIP}$ signal at $\beta$-actin (Actb), an AID non-targeted gene, is used as a negative control. The data represents the mean of the three independent experiments with standard deviations. Statistical significance as evaluated by Fisher's exact test $(\mathbf{c}, \mathbf{g})$ or Student's t-test $(\mathbf{h})$ is shown. The position of molecular weight markers is shown on the left of each western blot image. AIDER, AID fused with the hormone-binding domain of the estrogen receptor (ER); NS, not significant;

$\mathrm{OHT}$, 4-hydroxytamoxifen; SHM, somatic hypermutation.

(qPCR; Supplementary Fig. 7a). The ultraviolet-dependent incorporation of bTMP into genomic DNA was confirmed by a dot blot (Supplementary Fig. 7b). Using this method, we found that more bTMP was incorporated into the $S \mu$ region of TOP1-deficient P388 cells (P388/CPT45-GFP), compared with cells expressing exogenous TOP1 (P388/CPT45-GFP-TOP1), indicating that S-region DNA is more negatively supercoiled in TOP1-deficient cells (Fig. 6b). Similarly, SMARCA4 KD in CH12F3-2A cells also led to the enhanced accumulation of negative supercoils (Fig. 6c). These results further confirm the involvement of SMARCA4 in recruitment of TOP1, which suppresses the formation of non-B DNA structures by correcting uneven distribution of superhelix.

Next, we analyse the effect of SMARCA4 depletion on TOP1 recruitment and negative supercoiling at non-Igh AID target loci identified by whole-genome sequencing ${ }^{46-48}$. Among these; Pim1, $C d 83, C d 79 b, L y 6 e, I L 4 r a$ and $M y c$ are efficiently expressed in CH12-F3-2A cells (Fig. 7a). As shown in Fig. 7b, the depletion of 
a
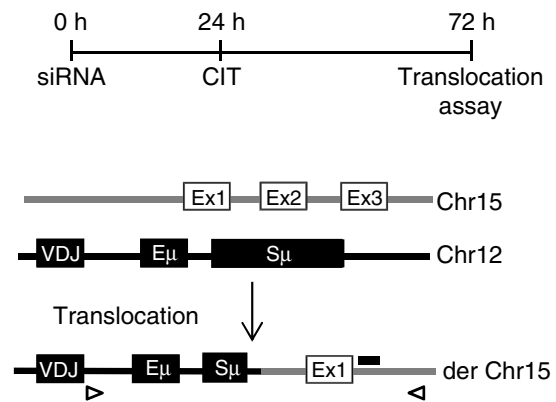

C
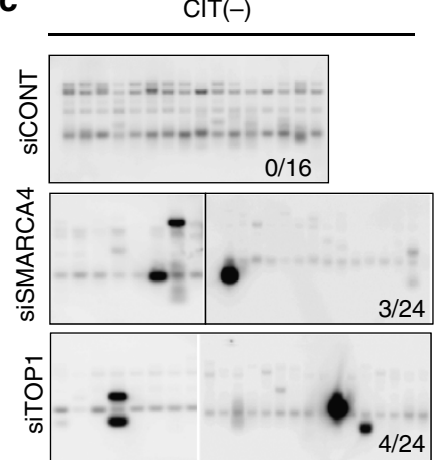

$\mathbf{e}$

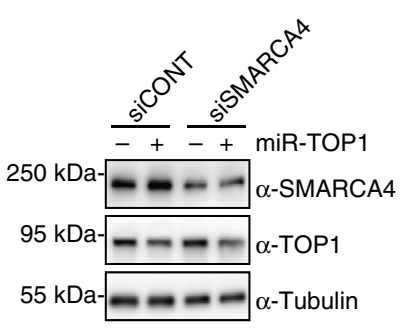

b

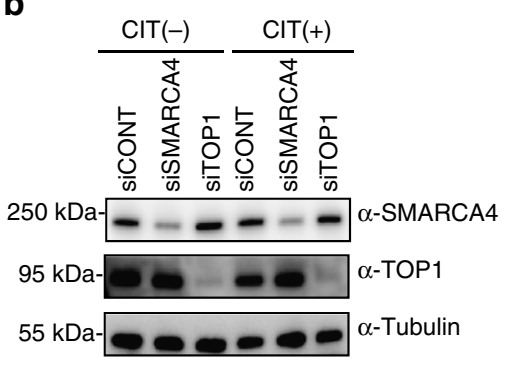

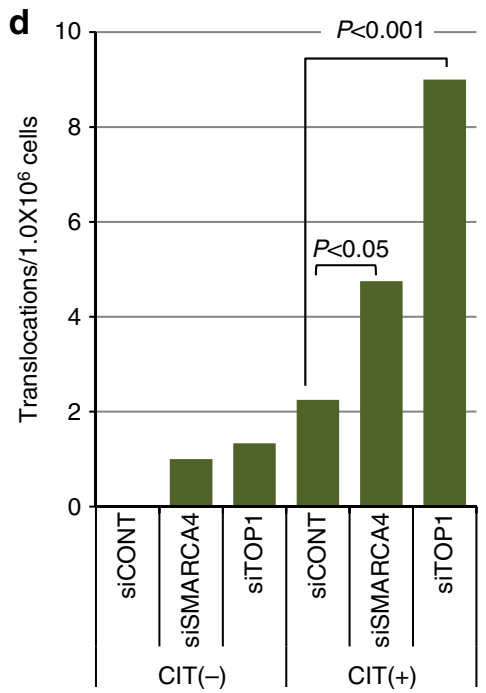

g

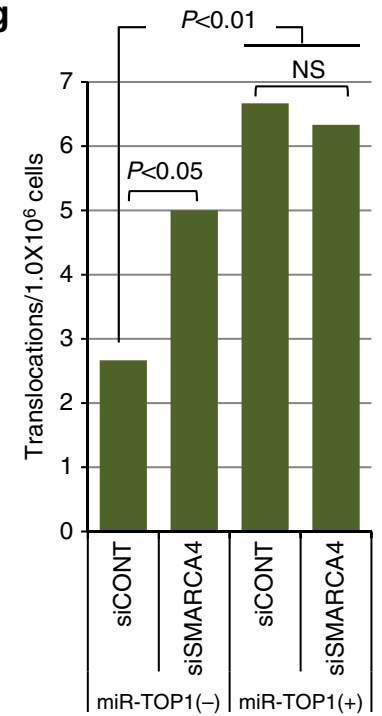

Figure 4 | SMARCA4 depletion augments chromosomal translocations in a TOP1-dependent manner. (a) Top: schematic illustration of experimental design for translocation assay in CH12F3-2A cells. Bottom: PCR amplification scheme to detect lgh/c-Myc chromosomal translocations. Triangles represent the positions of primers used to amplify the rearranged regions. The position of the Myc probe used in the Southern blot hybridization is shown as a horizontal black bar. (b) Confirmation of SMARCA4 or TOP1 KD by their respective siRNAs in $\mathrm{CH} 12 \mathrm{~F} 3-2 \mathrm{~A}$ cells used in the translocation assay shown in $\mathbf{c}$ and d. Expression of tubulin is shown as loading control. (c) Southern blot analysis of PCR-amplified fragments with Myc-specific probe. The CH12F3-2A cells were transfected with the indicated siRNAs and cultured with $(+)$ or without $(-)$ CIT stimulation. (d) Frequency of Igh/c-Myc chromosomal translocations derived from the indicated samples. (e) Confirmation of SMARCA4 or TOP1 KD in CH12F3-2A cells expressing Tet-inducible microRNA targeting Top1 mRNA (miR-TOP1). SMARCA4 or TOP1 were KD either by the transfection of the CH12F3-2A cells by SMARCA4 siRNA or by the treatment with tetracycline $(50 \mathrm{nM})$, respectively. The $(+)$ or $(-)$ signs indicate the presence or absence of Tet-inducible miR-TOP1. Expression of tubulin is shown as loading control. Genomic DNAs isolated from these cells were used for the translocation assays shown in $\mathbf{f}$ and $\mathbf{g}$. (f) Southern blot analysis of PCR-amplified fragments with Myc-specific probe. (g) Frequency of Igh/c-Myc chromosomal translocations derived from the SMARCA4- and/or TOP1-depleted $\mathrm{CH} 12 \mathrm{~F} 3-2 \mathrm{~A}$ cells. The numbers at bottom-right corners of the blots indicate the number of the translocations detected in total number of PCR reactions. Statistical significance as evaluated by Fisher's exact tests for indicated sets of data is shown. The position of molecular weight markers is shown on the left of each western blot image. CIT, CD4OL-IL4-TGF $\beta$; NS, not significant.

SMARCA4 by siRNAs resulted in the decrements in the TOP1 recruitment to these loci. Consistently, we found that SMARCA4 depletion leads to the increment in the incorporation of bTMP, a marker for an increment in negative supercoils (Fig. 7b). Interestingly, neither TOP1 recruitment nor bTMP incorporation was observed at a non-transcribed gene ( $T c r d)$, indicating a requirement of transcription for both TOP1 recruitment and accumulation of negative supercoils. Together, these data show that SMARCA4 is a general recruiter of TOP1, and required to maintain B- DNA structures.
As depletion of SMARCA4 caused reduction in TOP1 recruitment to non-Igh AID target loci, we analysed the formation DSBs at these loci using $\gamma \mathrm{H} 2 \mathrm{AX}-\mathrm{ChIP}$ assays. The depletion of SMARCA4 resulted in the increased DSBs at these loci (Supplementary Fig. 8a,b). Next, we investigated the H2AX phosphorylation by flow cytometry to assay the effect of SMARCA4 depletion on the global DSB formation. As shown in Supplementary Fig. 8c,d, we could not see large changes in the $\gamma \mathrm{H} 2 \mathrm{AX}$ level upon SMARCA4 depletion both in the absence or presence of AID, suggesting that TOP1 and 
a

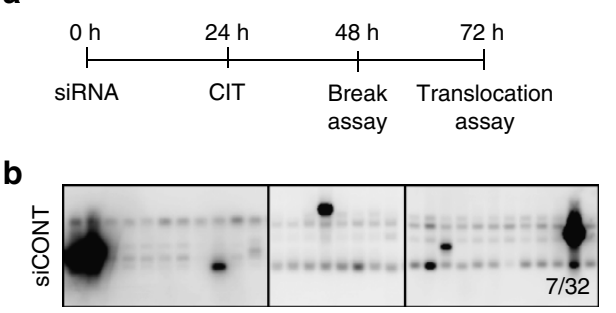

C

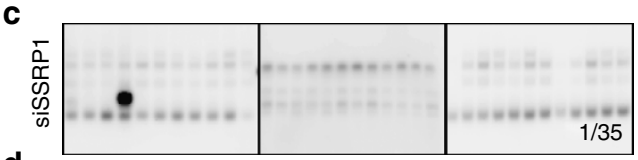

d

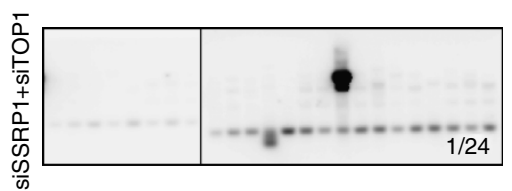

e

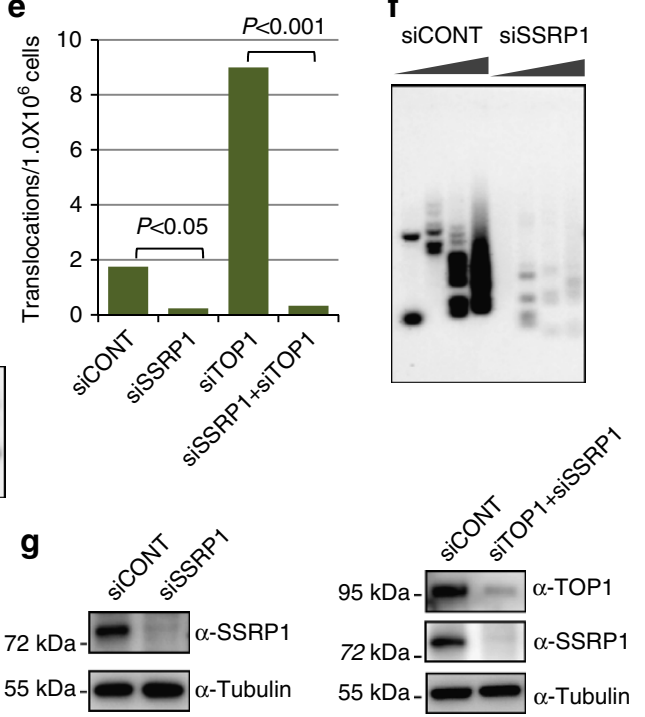

Figure 5 | FACT is essential for AID-induced genomic instability even in the TOP1-deficient cells. (a) Schematic illustration of experimental design for the analysis of DNA double-strand breaks by ligation mediated PCR (LM-PCR) and Igh/c-Myc chromosomal translocations in CH12F3-2A cells. (b-d) Southern blot analysis of PCR-amplified fragments with Myc-specific probe using genomic DNA prepared from $\mathrm{CH} 12 \mathrm{~F} 3-2 \mathrm{~A}$ cells transfected with the indicated siRNAs. The numbers at bottom-right corners of the blots indicate the number of the translocations detected in total number of PCR reactions. (e) Frequency of $\mathrm{Igh} / \mathrm{c}-\mathrm{Myc}$ chromosomal translocations derived from the indicated samples. The value of translocation frequency upon TOP1-KD is taken from Fig. 4c. (f) LM-PCR analysis of the AID-induced DNA double-strand breaks in the S $\mu$ region of CH12F3-2A cells following transfection with the indicated siRNAs. Wedges indicate a threefold increase in DNA amount. (g) Confirmation of single or simultaneous KD of SSRP1 and TOP1 in CH12F3-2A cells. Expression of tubulin is shown as loading control. Genomic DNAs isolated from these cells were used for the translocation assays and LM-PCR analysis. Statistical significance as evaluated by Fisher's exact test is shown. CIT, CD40L-IL4-TGF $\beta$.

transcription-dependent genomic damage is less frequent than those induced by DNA damage-inducing drugs. However, it is noteworthy that SMARCA4 KD almost doubled the percentage of cells with high level of $\gamma \mathrm{H} 2 \mathrm{AX}$ (Supplementary Fig. 8d).

ATPase activity of SMARCA4 is essential for TOP1 recruitment. To confirm the requirement of ATPase activity of SMARCA4 in TOP1 recruitment, we analysed the effect of two SMARCA4 point mutants: K785R (KR) and T910M (TM), both of which are incorporated normally into the BAF complex, but whose ATPase activity is highly compromised (Fig. 8a) ${ }^{49}$. First, we performed the CSR complementation assay by introducing siRNA-resistant GFP-tagged human wild-type (WT) or mutated SMARCA4 into CH12F3-2A cells, along with SMARCA4 siRNAs to deplete endogenous SMARCA4 (Fig. 8b). SMARCA4-targeted siRNA treatment decreased the expression of the endogenous SMARCA4, but not of the exogenous GFP-tagged SMARCA4 (Fig. 8c). CSR was rescued in cells expressing WT SMARCA4, but not in cells expressing the KR or TM mutant (Fig. 8b-d). Similarly, using TOP1-ChIP, we found that the defective TOP1 loading at the Igh locus in SMARCA4-depleted cells was significantly restored by expressing the siRNA-resistant WT SMARCA4, but not the KR or TM mutant (Fig. 8e). These results showed that the ATPase activity SMARCA4 is required for efficient CSR as well as for the recruitment of TOP1 to the Igh locus.

To know whether ATPase activity of SMARCA4 is also required for TOP1 recruitment to other loci, we performed TOP1-ChIP upon SMARCA4 depletion in CH12F3-2A cells pretreated by camptothecin (CPT), and found that SMARCA4 $\mathrm{KD}$ reduces the amount of TOP1 bound to chromatin at variety of tested loci even in the presence of CPT (Figs $7 \mathrm{~b}$ and $8 \mathrm{f}$ ). In addition, we also found that WT but not ATPase dead SMARCA4 proteins could rescue the defect in TOP1 recruitment in the presence of CPT (Fig. 8f). Taken together, these data suggest that
SMRACA4 ATPase activity is required for TOP1 trapping both in the absence (Fig. 8e) and presence of CPT (Figs 7b and 8f).

FACT is required for the association of TOP1 with H3K4me3. To examine the role of another TOP1-binding protein, $\mathrm{H} 3 \mathrm{~K} 4 \mathrm{me} 3$, in TOP1 recruitment, we performed peptide pulldown assays by incubating nuclear extracts of $\mathrm{CH} 12 \mathrm{~F} 3-2 \mathrm{~A}$ cells, which were prepared under native-IP conditions, with biotinylated histone $\mathrm{H} 3$ tail peptides carrying $\mathrm{H} 3 \mathrm{~K} 4 \mathrm{me} 3$ or other histone PTMs. Peptide-bound proteins were collected by streptavidin beads and subjected to western blotting (Fig. 9a). The results showed the preferential binding of TOP1 to H3K4me3 peptides, as compared with unmodified $\mathrm{H} 3, \mathrm{H} 3 \mathrm{~K} 9 \mathrm{me} 3$ or H3K27me3 peptides (Fig. 9a,b). Notably, each of the FACT subunits (SSRP1 and SPT16) showed relatively strong binding to H3K4me3 compared with the other peptides, indicating that TOP1, FACT and H3K4me3 are likely to form a complex (Fig. 9a,b). In contrast, SMARCA4 showed stronger binding to $\mathrm{H} 3 \mathrm{~K} 9 \mathrm{me} 3$ than $\mathrm{H} 3 \mathrm{~K} 4 \mathrm{me} 3$. To confirm the biological activity of the biotinylated histone peptides, the bound fractions were also tested for the presence of known histone-PTM readers, and shown to contain PHF8, HP1 $\alpha$ and SUZ12 in the complexes bound to $\mathrm{H} 3 \mathrm{~K} 4 \mathrm{me} 3, \mathrm{H} 3 \mathrm{~K} 9 \mathrm{me} 3$ or $\mathrm{H} 3 \mathrm{~K} 27 \mathrm{me} 3$, respectively ${ }^{50}$.

To further ascertain whether FACT is involved in binding of TOP1 to H3K4me3, we performed TOP1-IP from P388/CPT45GFP-TOP1 cells following siRNA-mediated depletion of SSRP1 or SMARCA4, and evaluated the association of TOP1 with H3K4me3 (Fig. 9c). As previously reported ${ }^{51}$, both SSRP1 and SPT16 proteins were significantly depleted by the transfection of siRNA targeting SSRP1 alone (Fig. 9c). Notably, TOP1-IP from FACT-depleted cells failed to pull down $\mathrm{H} 3 \mathrm{~K} 4 \mathrm{me} 3$, confirming that FACT is indeed essential for the binding of TOP1 to H3K4me3 (Fig. 9c). However, despite the absence of FACT, interaction of TOP1 with histone $\mathrm{H} 3$ and $\mathrm{H} 3.3$ remained intact, suggesting that FACT-independent TOP1 associations with other 
a

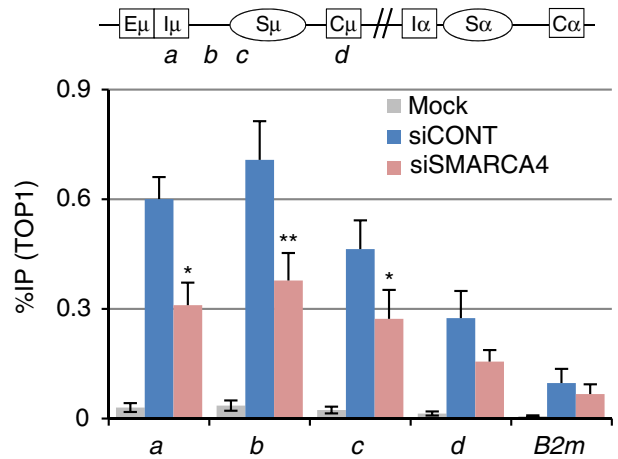

b

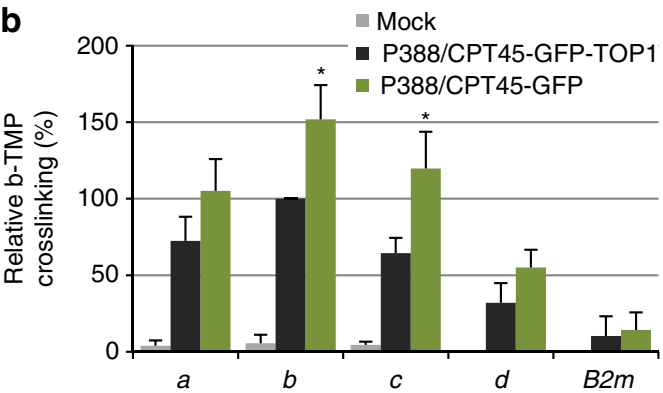

C

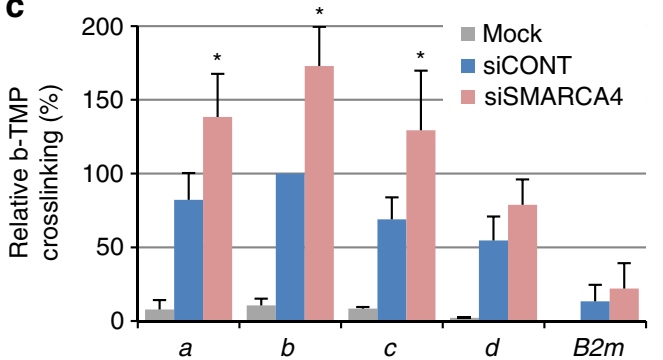

Figure 6 | SMARCA4 is required for efficient TOP1 recruitment to chromatin. (a) Schematic diagram showing the position of the ChIP assay PCR products in Igh locus. Bottom view shows the TOP1-ChIP assay following SMARCA4 KD in CH12F3-2A cells. Before lysis, the cells were pretreated with $50 \mathrm{nM}$ of bortezomib for $3 \mathrm{~h}$. ChIP data are normalized as in Fig. 3h, and represents the mean of the four independent experiments with standard deviations. $(\mathbf{b}, \mathbf{c})$ Analysis of negative superhelicity in P388/CPT45 cells expressing GFP or GFP-TOP1 (b), and CH12F3-2A cells after SMARCA4 KD (c). ChIP data is shown as the fraction of immunoprecipitated DNA (\%IP) normalized to the input DNA signals, and the maximum value in each data set was set as $100 \%$. The data represent the mean of the three independent experiments with standard deviations. The mock data show background values from control IP with no antibody. Asterisks ( ${ }^{\star}$ and ${ }^{\star \star}$ ) denote statistically significant differences with $P \leq 0.05$ and $P \leq 0.01$, respectively, as determined by Student's $t$-test. B2m, beta-2-microglobulin; b-TMP, biotin-trimethylpsoralen.

forms of chromatin also exist. In contrast to the effects of FACT $\mathrm{KD}$, SMARCA4 KD did not affect TOP1 binding to H3K4me3 or FACT. However, in the absence of FACT, the amount of SMARCA4 associated with TOP1 was decreased, suggesting that FACT may be involved in stabilizing the TOP1-SMARCA4 complex. We then analysed the ability of TOP1 to directly bind H3K4me3 using purified recombinant human TOP1 protein and customized histone tail peptide arrays. We found that TOP1 did not bind H3K4me3 directly, but showed a weak affinity for $\mathrm{N}$-terminal acetylated histone $\mathrm{H} 3$ and $\mathrm{H} 4$ peptides, confirming that binding of TOP1 to H3K4me3 was indirect (Supplementary Fig. 9).
Next, we performed TOP1-ChIPs to assess the effect of FACT $\mathrm{KD}$ on the recruitment TOP1 to the Igh locus in CH12F3-2A cells. In contrast to the drastic effect of SMARCA4 KD, FACT KD did not significantly affect TOP1 recruitment to the Igh locus (Fig. 9d). These results suggest that only a small fraction of the total TOP1 is associated with FACT and H3K4me3 at the Igh locus, although the majority of TOP1 is recruited by SMARCA4. Indeed, the interaction of TOP1 with Histone $\mathrm{H} 3$ and $\mathrm{H} 3.3$ remained intact even in the absence of FACT (Fig. 9c). Consistent with these findings, FACT KD did not cause any significant change in the accumulation of negative superhelicity (Fig. 9e). To the contrary, FACT appears to accumulate at non-B DNA containing chromatin as indicated by the increased chromatin binding of FACT upon TOP1 depletion (Fig. 9f). As FACT KD caused severe defects in AID-induced DNA breaks, it is likely that FACT recruits TOP1 to form the TOP1-FACT complex at non-B DNA containing chromatin, resulting in the irreversible DNA cleavage at non-B DNA (Fig. 9g). In fact, SSRP1 was originally identified as a protein recognizing a specific structure of DNA $^{52,53}$.

\section{Discussion}

Mutations or changes in the expression of SMARCA4 or other BAF complex subunits are associated with a wide variety of cancers, including lymphoid malignancies. Recent cancer genome sequencing studies showed that $\sim 20 \%$ of human cancers harbour mutations in at least one subunit of the BAF complex, suggesting a role of the SMARCA4-containing BAF complexes in the suppression of genomic instability ${ }^{34,35}$. However, the mechanisms linking the inactivation of BAF complex subunits with the induction of genomic instability are not well understood.

A recent study suggested that SMARCA4 is required for Topoisomerase- $2 \alpha$-mediated decatenation of the newly replicated sister chromatids during cell division ${ }^{49}$. On the other hand, the present study revealed the role of SMARCA4 in the efficient recruitment of TOP1 during transcription, and showed that SMARCA4 depletion mimics TOP1 deficiency in AID-induced genomic instability. Since TOP1 deficiency as well as defects in the processing of TOP1-DNA lesions are widely implicated in transcription-associated genomic instability and neurodegenerative diseases $^{3-5,16,18}$, it is possible that the genomic instability induced by SMARCA4 inactivation in human cancers is due, at least in part, to the compromised TOP1 recruitment to chromatin.

Recurrent chromosomal translocations involving $I g$ loci and proto-oncogenes such as $c-M Y C, B C L-2, B C L-6$ and FGFR are hallmarks of B-cell malignancies, and are associated with Burkitt's lymphoma, follicular lymphoma, diffuse large cell lymphoma and multiple myeloma, respectively ${ }^{54-56}$. We observed a dramatic increase in the frequency of chromosomal translocations involving Igh and $c-M y c$ loci in SMARCA4 or TOP1-deficient cells, indicating that defects in the efficient recruitment of TOP1 in SMARCA4-depleted cells induce genomic instability. Notably, even in the absence of AID activation, a low frequency of chromosomal translocations was observed in SMARCA4- or TOP1-depleted CH12F3-2A cells. In addition, SMARCA4 depletion also reduces the TOP1 occupancy and enhances negative supercoiling at non-Igh AID target loci. However, it remains to be studied whether depletion of SMARCA4 or TOP1 enhances mutations or chromosomal translocations involving these loci.

Although FACT and H3K4me3 also formed a complex with TOP1, their functions were distinct from that of SMARCA4. We found that in the absence of FACT, TOP1 failed to associate with H3K4me3, indicating that TOP1 association with the H3K4me3associated protein complex was FACT-dependent (Fig. 9c). In addition, we found that FACT depletion almost completely 


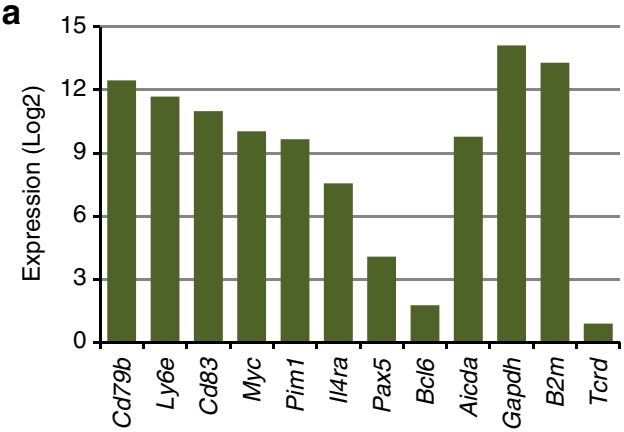

$c d 79 b$
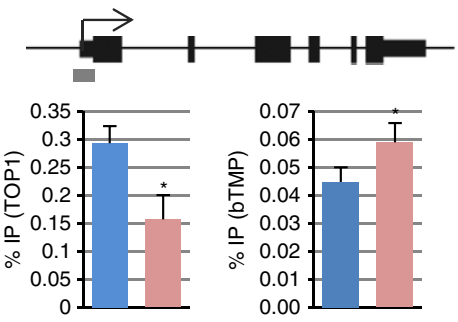

Cd83
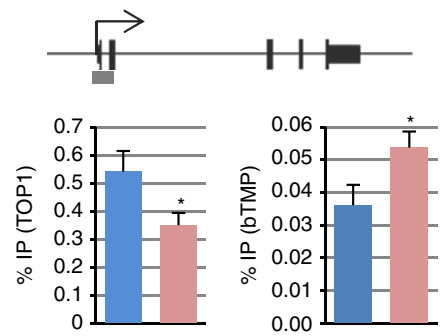

Gapdh
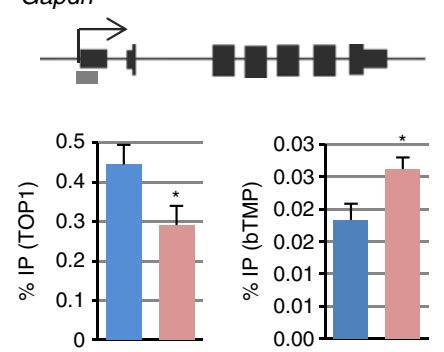

Myc

$B 2 m$ b

$\operatorname{lgh}$
siCONT
siSMARCA4
Positions of ChIP primers

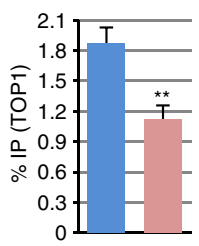

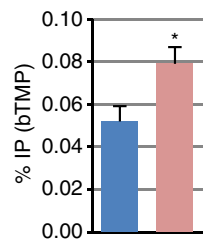

IL4ra
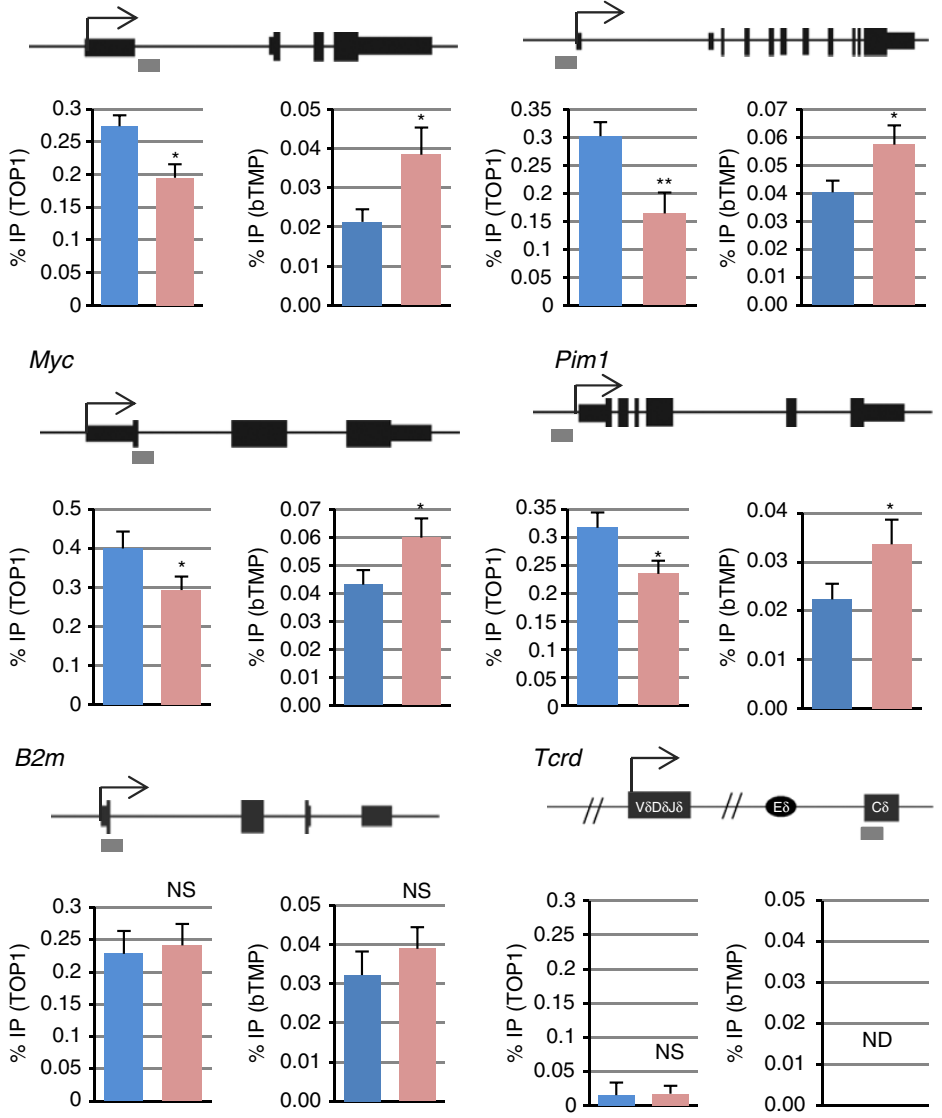

Figure 7 | SMARCA4 is required for TOP1 recruitment and maintenance of negative superhelicity at non-lgh targets of AID. (a) Expressions of non-Igh AID targets, Aicda, and AID non-targeted genes derived from microarray analysis of CIT stimulated CH12F3-2A cells. (b) Top: schematic diagram showing the position of the ChIP assay PCR products (underlined bar) in the respective Igh, non-Igh and AID non-targeted genes. Bottom: analysis of TOP1-ChIP (left) and negative superhelicity (right) following SMARCA4 KD in CH12F3-2AA cells. For TOP1-ChIP assay, before lysis, the cells were pretreated with $10 \mu \mathrm{M}$ of camptothecin for $30 \mathrm{~min}$. ChIP data are normalized as in Fig. 3h, and represent the mean of the three independent experiments with standard deviations. Asterisks ( ${ }^{*}$ and ${ }^{* \star}$ ) denote statistically significant differences with $P \leq 0.05$ and $P \leq 0.01$, respectively, as determined by Student's $t$-test. B2m, beta-2-microglobulin; Gapdh, glyceraldehyde-3-phosphate dehydrogenase; ND, not detected; NS, not significant; Tcrd, T-cell receptor delta chain.

abolished the generation of AID-induced $S$ region breaks as well as $I g h / c-M y c$ translocations. The strong inhibition of AID-induced breaks by FACT KD was probably due to the defect of TOP1 association with the H3K4me3-FACT complex, which may be involved in determining cleavage sites. Although FACT depletion disturbed the association of TOP1 with H3K4me3, it did not affect the binding of TOP1 with chromatin through $\mathrm{H} 3$ or H3.3. Thus, it is likely that FACT binds to only a small fraction of SMARCA4-recruited TOP1 to form complex with H3K4me3 probably at non-B DNA. Indeed, the depletion of TOP1, which induces the formation of non-B DNAs, resulted in the increased chromatin binding of FACT, indicating that FACT has an affinity for non-B DNAs (Fig. 9f). In fact, SSRP1, a subunit of FACT, was originally identified as a protein recognizing a specific DNA structure ${ }^{52,53}$. In contrast, SMARCA4 depletion suppressed the recruitment of TOP1 to chromatin, but had little effect on the association TOP1 with FACT and $\mathrm{H} 3 \mathrm{~K} 4 \mathrm{me} 3$. Thus, our findings showed that SMARCA4 is involved in the general recruitment of TOP1 to chromatin, while a limited amount of TOP1 recruited by SMARCA4 is sufficient to promote DNA cleavage through the interaction with FACT accumulated at the non-B DNA (Fig. 9f,g; Supplementary Table 7).

High transcriptional levels are associated with mutations, deletions, recombination and other deleterious genetic events that 
a

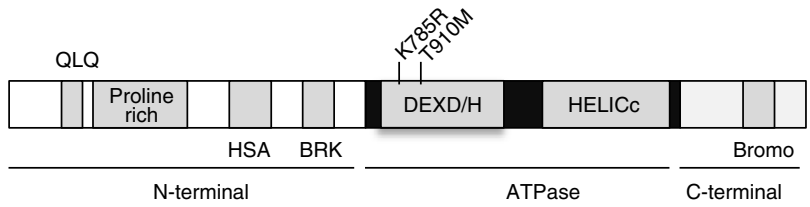

b

$\begin{array}{ccc}\text { SiRNA } & 24 \mathrm{~h} & 54 \mathrm{~h} \\ \text { plasmid } & \text { CIT } & \text { CSR assay } \\ & & \text { TOP1-ChIP }\end{array}$

c

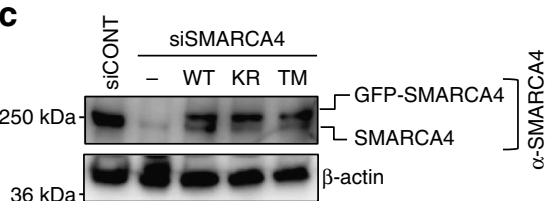

d

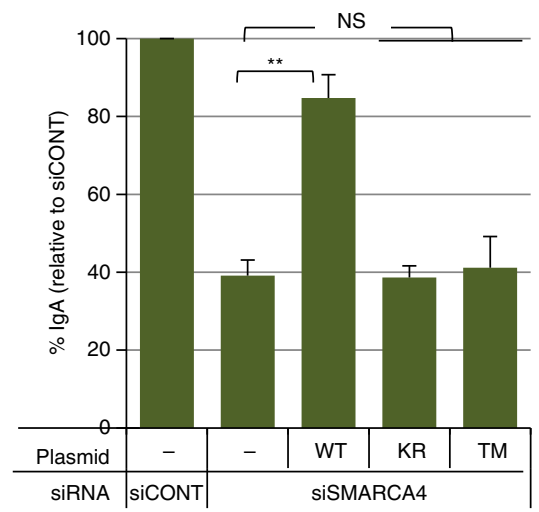

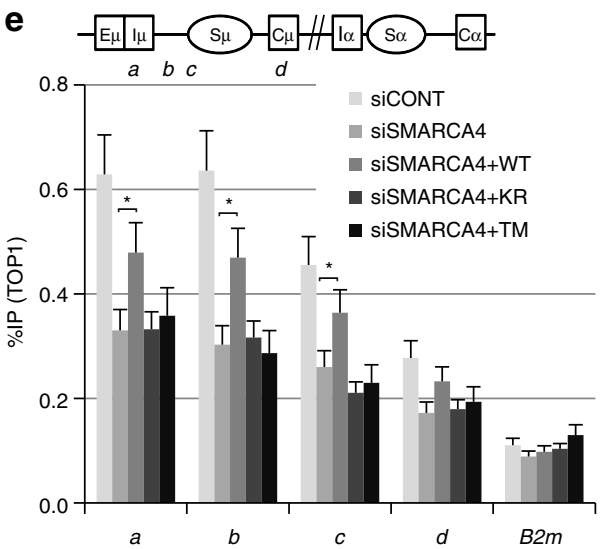

f

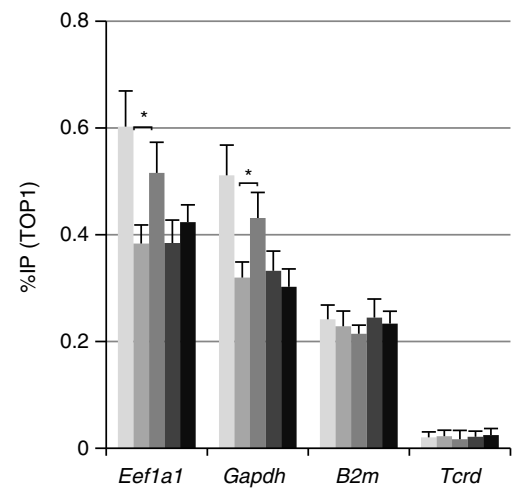

Figure 8 | ATPase activity of SMARCA4 is essential for efficient TOP1 recruitment. (a) Top: schematic representation of the human SMARCA4 protein showing the positions of the mutations in the analysed mutants. Bottom: schematic representation of GFP-fused WT- or mutated-SMARCA4 proteins. (b) Schematic representation of the experimental design for the CSR complementation and TOP1-ChIP assays. (c) Confirmation of the siRNA-mediated KD of endogenous mouse SMARCA4, and expression of GFP-tagged human SMARCA4 (WT, and KR and TM mutants). Expression of $\beta$-actin is shown as loading control. (d) CSR complementation assay using CH12F3-2A cells expressing siRNA-resistant human SMARCA4. Data are presented as \% IgA switching relative to the IgA switching in cells transfected with control siRNA (siCONT) with similar GC content. The data represent the mean of the three independent experiments with standard deviations. (e,f) TOP1-ChIP assays following CSR complementation in CH12F3-2A cells. Before lysis, the cells were pretreated with either $50 \mathrm{nM}$ of bortezomib for $3 \mathrm{~h} \mathrm{(e)} \mathrm{or} 10 \mu \mathrm{M}$ of camptothecin for $30 \mathrm{~min}(\mathbf{f})$. Top view shows schematic diagram with the position of the ChIP assay PCR products in Igh locus. ChIP data were normalized as in Fig. 3h, and represent the mean of the three independent experiments with standard deviations. Asterisks ( ${ }^{\star}$ and ${ }^{\star *}$ ) denote statistically significant differences with $P \leq 0.05$ and $P \leq 0.01$, respectively, as determined by Student's $t$-test. The position of molecular weight markers is shown on the left of each western blot image. B2m, beta-2-microglobulin; CIT, CD4OL-IL4-TGF $\beta$; CSR, class switch recombination; Eef1al, eukaryotic translation elongation factor 1 alpha 1; Gapdh, glyceraldehyde-3-phosphate dehydrogenase; NS, not significant; Tcrd, T-cell receptor delta chain.

can threaten genomic stability ${ }^{5,57}$. TOP1 and transcriptioninduced non-B DNA structures formed at repetitive or palindromic sequences are involved in transcription-associated mutagenesis, unstable triplet repeat diseases and AID-induced Ig gene diversification ${ }^{11-13,23,24}$. In addition, there is evidence that FACT and $\mathrm{H} 3 \mathrm{~K} 4 \mathrm{me} 3$ are involved in AID-induced genetic alterations ${ }^{25,29,30}$. Here, we showed that KD of TOP1 or SMARCA4 increased the accumulation of negative supercoils, and promoted $\mathrm{V}$ and $\mathrm{S}$ region DNA cleavage, resulting in increased frequencies of SHM and $I g h / c-M y c$ chromosomal translocations. Notably, the simultaneous depletion of SMARCA4 and TOP1 did not show additive effects on SHM or $I g h / c-M y c$ translocations, suggesting that the functions of these two factors may overlap. Taken together, these results suggest that the SMARCA4 KD-mediated augmentation of SHM or Igh/c-Myc translocations was due to inefficient TOP1 recruitment to the $S$ and $M y c$ regions. In addition, we showed that the ATPase activity of SMARCA4, which is essential for chromatin remodelling, was required for efficient TOP1 recruitment.

The accumulation of unwound DNA in SMARCA4- or TOP1-depleted cells increased the frequency of $I g h / c-M y c$ translocations, even in the absence of AID induction, suggesting that the excessive accumulation of non-B DNA promotes DNA cleavage. On the other hand, the depletion of FACT, which was required for $\mathrm{H} 3 \mathrm{~K} 4 \mathrm{me} 3$ marking and for the association of TOP1 with $\mathrm{H} 3 \mathrm{~K} 4 \mathrm{me} 3$ did not affect the accumulation of non-B DNA, but completely abolished the AID-induced DNA cleavage and $I g h / c-M y c$ translocations. Notably, TOP1 KD, which normally results in the accumulation of non-B DNA structures, failed to induce $I g h / c-M y c$ translocations in FACT-depleted cells (Fig. 5a-g). These data suggest that the accumulation of non-B DNA structures promotes AID-induced DNA cleavage only in the presence of FACT. Taken together, these observations suggest that AID-induced genetic alterations and transcriptiondependent genomic instability share a common mechanism involving the accumulation of non-B DNA and the recruitment of TOP1 to chromatin, which is mediated by SMARCA4, FACT and $\mathrm{H} 3 \mathrm{~K} 4 \mathrm{me} 3$.

In conclusion, we showed that TOP1 is recruited by SMARCA4 to chromatin so that transcription-associated uneven distribution of superhelix is corrected. A small fraction of TOP1 binds to FACT that is enriched at non-B DNA containing chromatin, and forms a complex with $\mathrm{H} 3 \mathrm{~K} 4 \mathrm{me} 3$, which is essential for AID-induced DNA cleavage (Fig. 9g). Depletion of 
FACT dissociates TOP1 from $\mathrm{H} 3 \mathrm{~K} 4 \mathrm{me} 3$-associated complex, and abolishes AID-induced DNA cleavage. Deficiency of either TOP1 or SMARCA4 reduces TOP1 binding to chromatin, and promotes the formation of non-B DNA structures, but a small amount of TOP1 can still bind to FACT and H3K4me3 at non-B DNA containing chromatin, and promotes genomic instability.

\section{Methods}

Cell lines and RNAi oligonucleotide transfection. The human Burkitt's lymphoma line BL2, the mouse B-cell lymphoma line $\mathrm{CH} 12 \mathrm{~F} 3-2 \mathrm{~A}$ expressing $\mathrm{Bcl} 2$, and the TOP1-deficient P388/CPT45 cells expressing GFP (P388/CPT45-GFP) or GFPtagged human TOP1 (P388/CPT45-GFP-TOP1) were previously described $24,29,44$ The BL2 and P388/CPT45 cells also express AIDER, a fusion protein in which AID is fused to the hormone-binding domain of the estrogen receptor (ER), which is activated by treating cells with OHT. Chemically modified Stealth siRNA a

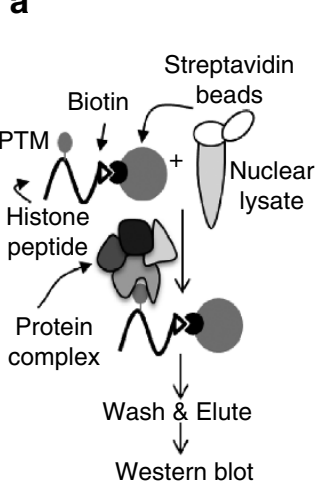

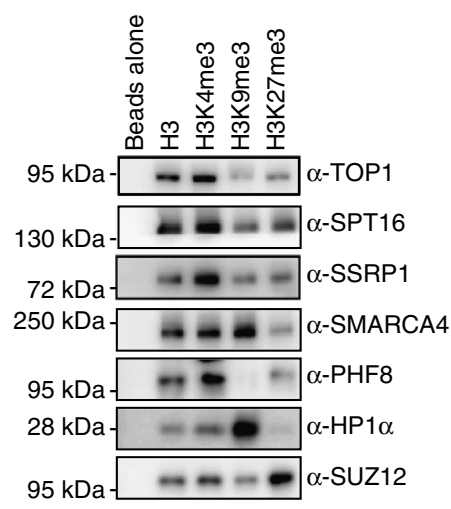

b

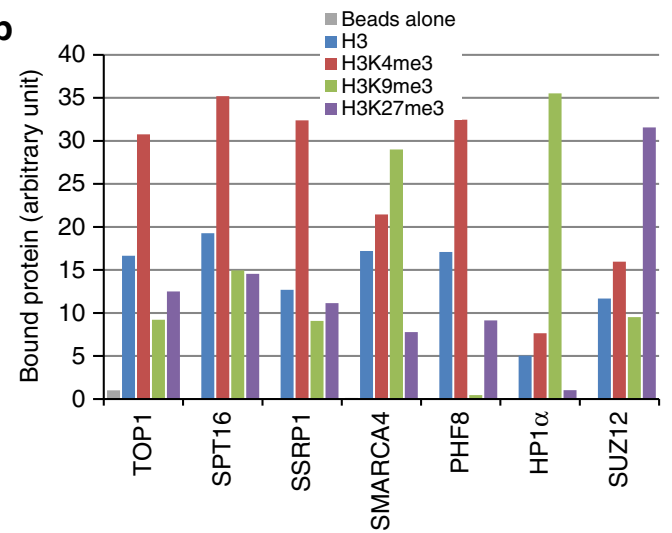

e

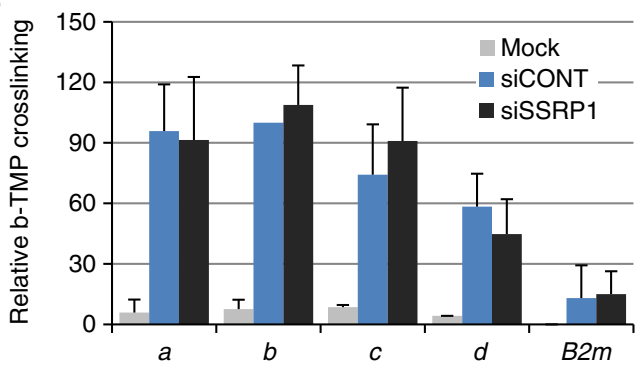

C

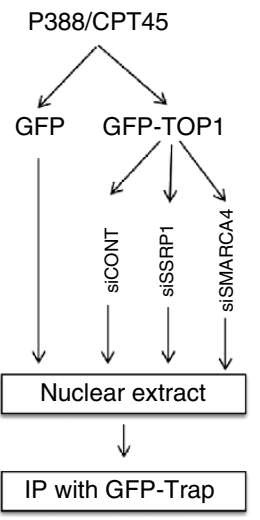

$\frac{\text { Input }}{\text { GFP-TOP1 }} \frac{\text { IP }}{\text { GFP-TOP1 }}$

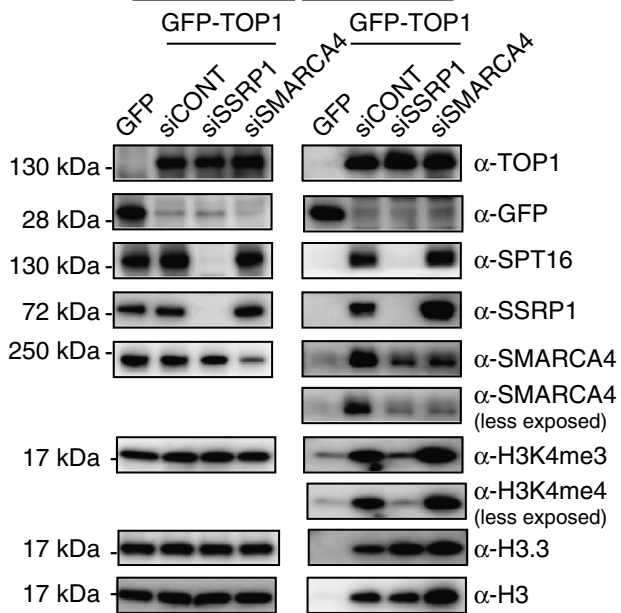

d

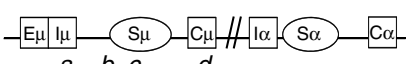
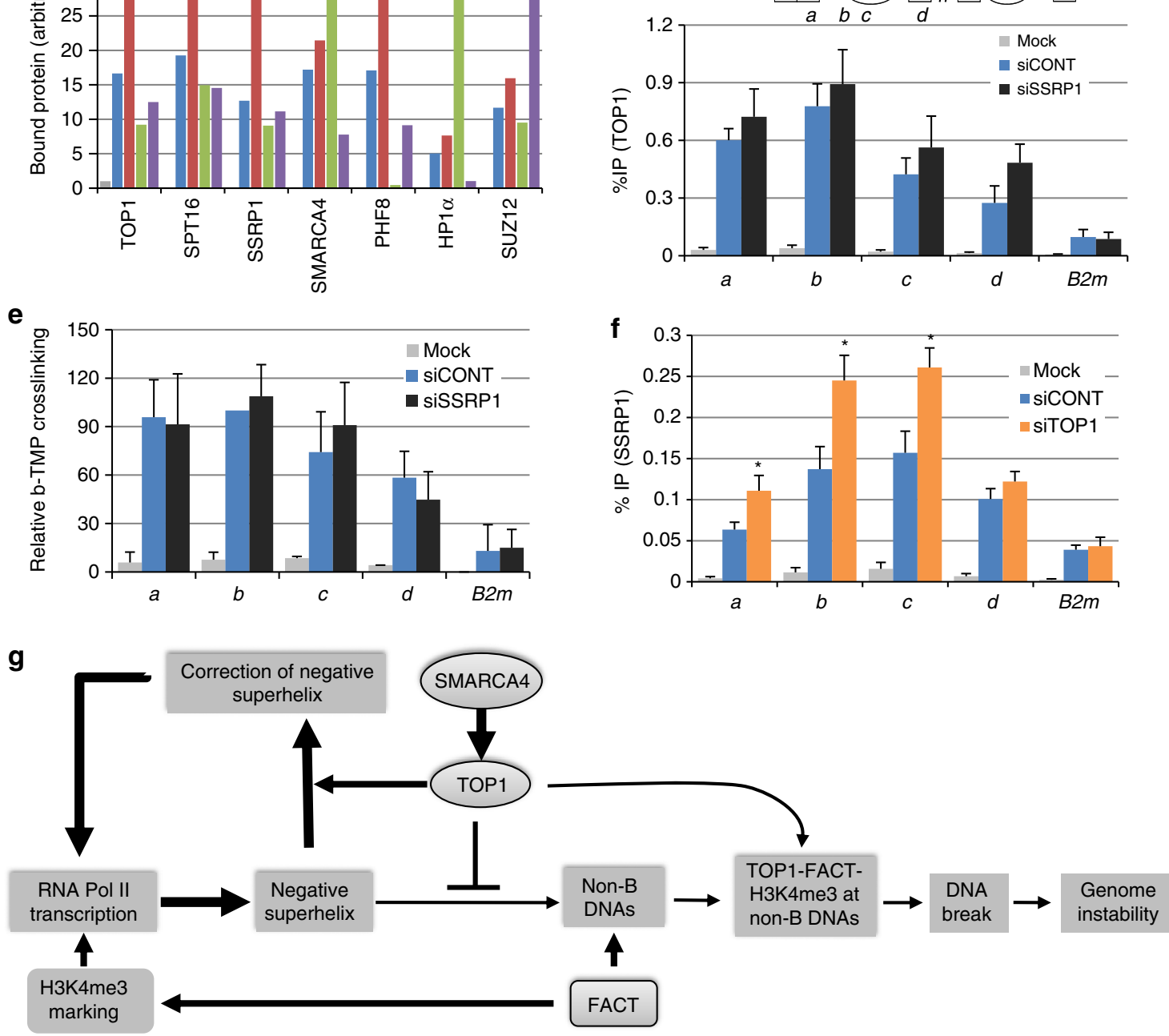
oligonucleotides (Invitrogen) were introduced into cells using the Nucleofector 96-well electroporation system (Lonza) to knockdown the expression of specific genes. After electroporation, the cells were cultured for $24 \mathrm{~h}$, and then stimulated by CIT (CD40L, IL4 and TGF $\beta$ ) or OHT $(1 \mu \mathrm{M})$, and cultured for another $24-72 \mathrm{~h}$ before collection and analysis. The list of antibodies, primers and Stealth siRNAs used in this study is shown in Supplementary Tables 8-10.

Analysis of CSR and SHM. CH12F3-2A cells were CIT-stimulated for $24-48 \mathrm{~h}$ to induce CSR, and surface expression of IgM and IgA was analysed by staining with FITC-conjugated anti-mouse IgM (eBioscience) and PE-conjugated anti-mouse IgA (Southern Biotechnology Associates) antibodies, respectively. Flow cytometry analysis were performed using a Becton-Dickinson FACS-Calibur, as previously described $^{30}$. Analysis of CSR in NIH 3T3 cells expressing artificial switch substrate SCI $(\mu, \alpha)$ and AIDER was performed as described earlier ${ }^{42}$. Briefly, NIH 3 T3 cells were transfected with control siRNA or human SMARCA4 siRNA using Lipofectamine 2000 (Invitrogen). Twenty-four hours post transfection, cells were stimulated with $1 \mu \mathrm{M}$ of OHT for $48 \mathrm{~h}$, and stained with allophyco-cyaninconjugated anti-CD $8 \alpha$ (eBioscience)

To analyse the effect of SMARCA4 depletion on SHM in BL2 cells expressing AIDER, the cells were transfected with SMARCA4 siRNA, cultured for $24 \mathrm{~h}$ and then treated with OHT $(1 \mu \mathrm{M})$ for $72 \mathrm{~h}$, followed by total RNA extraction using TRIzol (Invitrogen). The cDNA was synthesized from $0.5-1 \mu \mathrm{g}$ of total RNA using Oligo d(T)-primers and SuperScript III (Invitrogen). PCR fragments corresponding to the 426-bp rearranged $\mathrm{V}_{\mathrm{H}} 4-39-\mathrm{J}_{\mathrm{H}} 5$ region were cloned into the pGEM-T Easy Vector (Promega). The 408-bp sequence between the two primers was analysed for the presence of mutations ${ }^{58}$. Analysis of somatic mutations in the S-region of the P388/CPT45 cells was performed as previously described ${ }^{24}$. The mutation frequency was calculated as the number of mutations per total bases from independent experiments. The primers used for SHM analysis are shown in Supplementary Table 8 .

Co-immunoprecipitation. TOP1-deficient mouse B-cell lymphoma cell line P388/CPT45 expressing AIDER, and either GFP or GFP-TOP1 was used to co-IP TOP1-associated proteins. Chemical crosslinking-based IP (DSP-IP) was performed using DSP (Thermo Scientific) ${ }^{59}$. Cells $\left(2 \times 10^{8}\right)$ were incubated in phosphate-buffered saline (PBS) containing $0.6 \mathrm{mM}$ DSP for $30 \mathrm{~min}$ at $4{ }^{\circ} \mathrm{C}$ with mild rotation. After removing PBS, the remaining DSP was quenched by incubating the cells in TBS (50 mM Tris-Cl pH 7.4, $150 \mathrm{mM} \mathrm{NaCl}, 1 \mathrm{mM}$ EDTA) for $15 \mathrm{~min}$ on ice. To prepare nuclear extracts, cells were subjected to hypotonic lysis, and then the isolated nuclei were lysed in lysis buffer ( $50 \mathrm{mM}$ Tris- $\mathrm{Cl} \mathrm{pH} \mathrm{7.5,}$ $150 \mathrm{mM} \mathrm{NaCl}, 0.25 \%$ Triton X-100, $0.25 \%$ Na-deoxycholate, $0.05 \%$ SDS, $1 \mathrm{mM}$ EDTA, $5 \%$ glycerol) containing $5 \mathrm{mM} \mathrm{MgCl}_{2}, 500 \mathrm{U} \mathrm{ml}^{-1}$ of Benzonase nuclease (Novagen), $300 \mathrm{mM} \mathrm{NaCl}$, and protease inhibitor cocktail (Roche). The nuclear extract was then sonicated for $10 \mathrm{~min}$, followed by rotation for $1 \mathrm{~h}$ at $4{ }^{\circ} \mathrm{C}$ and centrifugation at $20,000 \mathrm{~g}$ for $10 \mathrm{~min}$. The supernatant was pre-cleared with blocked agarose beads (Chromotek) and then incubated with GFP-Trap_A (Chromotek) for $2 \mathrm{~h}$ at $4^{\circ} \mathrm{C}$. The agarose beads were washed sequentially with TBS, lysis buffer, high-salt lysis buffer $(500 \mathrm{mM} \mathrm{NaCl})$ and TBS. Finally, the beads were re-suspended in $100 \mu \mathrm{l} 2 \times$ SDS-sample buffer containing $5 \% \beta$-mercaptoethanol, and heated for $10 \mathrm{~min}$ at $95^{\circ} \mathrm{C}$ to elute the bound proteins.

To perform IP without crosslinking (native-IP), the nuclei prepared from $2 \times 10^{8}$ cells were suspended in HGN165 buffer (20 mM HEPES pH 7.4, $10 \%$ glycerol, $165 \mathrm{mM} \mathrm{NaCl}$ ) containing $2 \mathrm{mM} \mathrm{MgCl} 2,1 \mathrm{mM} \mathrm{DTT}, 500 \mathrm{U} \mathrm{ml}^{-1}$ of Benzonase nuclease, $0.1 \%$ Triton X-100 and protease inhibitor cocktail, followed by mild sonication to rupture the nuclei and rotation at $4{ }^{\circ} \mathrm{C}$ for $1 \mathrm{~h}$. After collecting the supernatant, the insoluble pellet was sequentially extracted with the abovementioned buffer containing a higher concentration of Triton X-100 (0.3\%), followed by extraction with buffer containing a higher concentration of $\mathrm{NaCl}$ (200 and $250 \mathrm{mM}$ ). The four extracts were pooled, centrifuged and the supernatants were IPed using GFP-Trap_A. The beads were washed extensively with HGN165 buffer, and the bound fractions were eluted as described above. To perform reverse co-IP, HEK 293T cells were transfected with $10 \mu \mathrm{g}$ of pEGFP-C2-SSRP1 or pEGFP-C2-SMARCA4 plasmids, and $48 \mathrm{~h}$ post transfection, the cells were collected and nuclear extracts were IPed as described for native-IP. The eluted proteins were subjected to western blotting with specific antibodies. Less-cropped western blots are shown in Supplementary Fig. 10.

Proteomic analysis. For quantitative analysis of crosslinked IP samples, ${ }^{18} \mathrm{O}$ postlabelling method was used ${ }^{60}$. Briefly, $130 \mu \mathrm{g}$ samples obtained from the crosslinked IP experiments were digested with 1:50 modified porcine trypsin (Promega) in the presence of $8 \mathrm{M}$ urea and $100 \mathrm{mM}$ Tris- $\mathrm{HCl}(\mathrm{pH} 8.0)$ after reduction with $2 \mathrm{mM}$ dithiothreitol and alkylation with $10 \mathrm{mM}$ iodoacetamide. The reaction was stopped with $1 \%$ TFA, and cleaned using a C18 cartridge (Bond Elute C18 EWP, Agilent). The eluted peptides were divided and dried completely in a Speed-Vac concentrator (Thermo Fisher). One half of the dried peptides were labelled with ${ }^{18} \mathrm{O}$ and the rest with ${ }^{16} \mathrm{O}$ by incubating with 1:50 trypsin/peptide $(\mathrm{w} / \mathrm{w})$ in $100 \mu \mathrm{l}$ of $50 \mathrm{mM} \mathrm{NH}_{4} \mathrm{HCO}_{3}$ buffer made either in $\mathrm{H}_{2}^{18} \mathrm{O}$ or in $\mathrm{H}_{2}^{16} \mathrm{O}$ containing $10 \mathrm{mM}$ $\mathrm{CaCl}_{2}$. After incubating for $5 \mathrm{~h}$ at $37^{\circ} \mathrm{C}$ with shaking, the reaction was stopped by boiling the sample in a $100^{\circ} \mathrm{C}$ water bath for $20 \mathrm{~min}$, followed by immediate freezing at $-80^{\circ} \mathrm{C}$. Equal amounts of TOP1 and control IP samples were mixed and fractionated over a strong cation exchange column ${ }^{61}$. To compensate for the incomplete labelling, TOP1 sample was labelled with ${ }^{18} \mathrm{O}$ and control sample was labelled with ${ }^{16} \mathrm{O}$ in one sample set, while the labelling was reversed in the second set. Twenty-two fractions obtained were separately subjected to the LC-MS/MS (liquid chromatography tandem mass spectrometry) analysis, using nano high-performance liquid chromatography (LC Packings, UltiMate 3000) interfaced online to a hybrid Fourier-transform ion cyclotron resonance mass spectrometer (Thermo Fisher, LTQ-FT). The details of the analytical conditions were as described previously ${ }^{6}$. The data were processed using a Mascot Distiller software (ver. 2.3.2, Matrix Science, UK), and subjected to database search (Mascot, version 2.3, Matrix Science) against Mus musculus protein sequences in the Swiss-Prot database. Mascot distiller was also used for the quantification of proteins.

Owing to the limited amounts of samples obtained, semi-quantitative analysis was used for native-IP experiments. Briefly, $30 \mu \mathrm{g}$ of immunoprecipitated protein samples were electrophoresed on SDS-PAGE gels (4-20\% acrylamide gel), and the gel was stained with Coomassie brilliant blue. Each lane was cut into 15 slices of equal size, destained and subjected to in-gel digestion with trypsin as described previously ${ }^{63}$. The obtained peptides were subjected to LC-MS/MS analyses as described above.

Histone peptide pull-down assay. The nuclear extracts for histone peptide pull-down (PPD) assays were prepared as described for native-IP. For PPD assay, $40 \mu \mathrm{l}$ of streptavidin Dynabeads M-280 (Invitrogen) were coupled to $20 \mu \mathrm{g}$ of biotinylated histone peptides and then incubated with a nuclear extract equivalent to $2 \times 10^{7}$ cells for $2 \mathrm{~h}$ at $4{ }^{\circ} \mathrm{C}$. The magnetic beads were washed extensively with HGN165 buffer, and peptide-bound proteins were eluted by heating the beads in $100 \mu \mathrm{l}$ of $2 \times$ SDS-sample buffer containing $5 \% \beta$-mercaptoethanol at $95^{\circ} \mathrm{C}$ for 10 min. The eluted proteins were subjected to western blotting with specific antibodies. Information about the peptides used for PPD assay is shown in Supplementary Table 11 .

Analysis of Igh/c-Myc chromosomal translocations. The $I g h / c-M y c$ translocation junctions (derivative chromosome 15) were PCR-amplified from genomic

Figure 9 | FACT is required for TOP1-H3K4me3 complex formation. (a) Histone peptide pull-down assays using biotinylated peptides carrying either H3K4me3 or other histone PTMs. Cell lysates for peptide pull-down assays from CIT-stimulated CH12F3-2A cells were prepared as described for native-IP (see 'Methods'). Pulled-down proteins were immunoblotted with the indicated antibodies. (b) Densitometric quantification of western blot images shown in Fig. 9a is performed to analyse relative binding of the indicated proteins with the histone peptides. (c) TOP1 co-IP following SSRP1 or SMARCA4 KD in P388/CPT45-GFP-TOP1 cells. Cell lysates prepared form P388/CPT45-GFP-TOP1 cells transfected with the indicated siRNAs were subjected to immunoprecipitation with GFP-Trap_A, and the IPed proteins were western blotted with the indicated antibodies. (d) TOP1-ChIP analysis following SSRP1 $\mathrm{KD}$ in $\mathrm{CH} 12 \mathrm{F3}-2 \mathrm{~A}$ cells. Before lysis, the cells were pretreated with $50 \mathrm{nM}$ of bortezomib for $3 \mathrm{~h}$. ChIP data were normalized as in Fig. $3 \mathrm{~h}$. The data represent the mean of the three independent experiments with standard deviations. The mock data show background values from control IP with no antibody. (e) Analysis of negative superhelicity in the SSRP1-depleted cells. ChIP data were normalized as in Fig. 6c. (f) Analysis of SSRP1-ChIP following TOP1 KD in $\mathrm{CH} 12 \mathrm{~F} 3-2 \mathrm{~A}$ cells. ChIP data are normalized as in Fig. 3h, and represent the mean of the three independent experiments with standard deviations. Asterisks ${ }^{\star}{ }^{\star}$ ) denote statistically significant differences with $P \leq 0.05$, as determined by Student's $t$-test. The position of molecular weight markers is shown on the left of each western blot image. B2m: beta-2-microglobulin; b-TMP, biotin-trimethylpsoralen. (g) Diagrammatic representation of role of SMARCA4, FACT and H3K4me3 in TOP1-mediated genomic instability. High levels of transcription lead to the accumulation of negative superhelix. SMARCA4 recruits TOP1 to actively transcribed chromatin to correct excessive accumulation of negative superhelix and prevent the formation of non-B DNAs at repetitive sequences. Reduction in TOP1 recruitment causes accumulation of negative superhelix leading to the formation of non-B DNA structures at repetitive sequences. On the other hand, FACT binds to non-B DNA through SSRP1, and traps a fraction of SMARCA4-recruited TOP1, which facilitates the formation of a complex of TOP1, FACT and H3K4me3 at non-B DNA containing chromatin, resulting in the DNA cleavage. 
DNA obtained from $48 \mathrm{~h}$ CIT-stimulated CH12F3-2A cells using the Expand Long Template PCR System ${ }^{64}$. A total of 16 to 48 aliquots of genomic DNA were analysed in separate reactions. The conditions for both first and second round of PCR were as follows: $94^{\circ} \mathrm{C}$ for $3 \mathrm{~min}$, followed by 25 cycles at $94{ }^{\circ} \mathrm{C}$ for $15 \mathrm{~s} ; 62^{\circ} \mathrm{C}$ for $15 \mathrm{~s} ; 68^{\circ} \mathrm{C}$ for $7.5 \mathrm{~min}$ and a final extension of $5 \mathrm{~min}$ at $68^{\circ} \mathrm{C}$. The PCR products were electrophoresed on ethidium bromide-containing $1 \%$ agarose gels and subjected to Southern blotting with $M y c$-specific probe. The primer and probe sequences are shown in Supplementary Table 8.

Analysis of DNA double-strand breaks. Genomic DNAs were prepared from CH12F3-2A cells stimulated with CIT for $24 \mathrm{~h}$. DSBs were analysed by ligation-mediated PCR as previously described ${ }^{65,66}$. Briefly, living CH12F3-2A cells were isolated by Percoll density gradient centrifugation and embedded in low-melting-point agarose followed by DNA extraction within low-melting agarose plugs. A total of $20 \mu \mathrm{l}$ of genomic DNA was ligated with linker in a final volume of $100 \mu \mathrm{l}$. Ligation reaction was stopped by adding $200 \mu \mathrm{l}$ of $\mathrm{H}_{2} \mathrm{O}$ followed by heating at $70^{\circ} \mathrm{C}$ for $10 \mathrm{~min}$. Threefold dilutions of input DNA were PCR amplified by KOD-FX-Neo polymerase (TOYOBO). The amount of ligated DNA in the input was normalized with Gapdh DNA. PCR products were electrophoresed on $1 \%$ agarose gels followed by Southern blotting with $5^{\prime} \mathrm{S} \mu$ probe. The primer and probe sequences are shown in Supplementary Table 8.

Chromatin immunoprecipitation. The $\gamma \mathrm{H} 2 \mathrm{AX}-\mathrm{ChIP}$ assay was performed using ChIP-IT Express Kit (Active Motif) as previously described ${ }^{29}$. For TOP1-ChIP, CIT-stimulated CH12F3-2A cells $\left(3 \times 10^{6}\right)$ were treated with either $50 \mathrm{nM}$ of Bortezomib (Janssen Pharmaceuticals) for $3 \mathrm{~h}$ or $10 \mu \mathrm{M}$ of camptothecin (Calbiochem) for $30 \mathrm{~min}$. The cells were rapidly lysed in $0.9 \mathrm{ml}$ of $10 \mathrm{mM}$ Tris-Cl pH6.8, $20 \mathrm{mM}$ EDTA, 4\% Triton X-100, 6M guanidinium isothiocyanate, $1 \%$ Sarkosyl and $60 \mathrm{mM}$ DTT. The genomic DNA was precipitated by addition of $0.6 \mathrm{ml}$ of $100 \%$ ethanol followed by centrifugation ${ }^{67}$. The genomic DNA was sheared using components ChIP-IT Express Kit (Active Motif), and $20 \mu \mathrm{g}$ of genomic DNA was immunoprecipitated with $3 \mu \mathrm{g}$ of TOP1 antibody and Protein G-coupled magnetic beads (Active Motif). The beads were sequentially washed by a standard series of wash buffers (low salt, high salt, $\mathrm{LiCl}$ and Tris/EDTA), and the IPed chromatin was eluted with $1 \%$ SDS and $0.1 \mathrm{M} \mathrm{NaHCO}_{3}$ and purified using phenol-chloroform. The locus-specific enrichment was detected by $\mathrm{qPCR}$, and normalized to the input DNA. The primers and antibodies used are shown in Supplementary Tables 8 and 9, respectively.

Analysis of negative superhelicity. To identify regions of negative superhelicity, we partially adapted a biotin-trimethylpsoralen (bTMP)-based assay described elsewhere ${ }^{68}$. Biotin-TMP was synthesized by the biotinylation of $4^{\prime}$-aminomethyltrioxsalen (Sigma). Briefly, cells were treated with $30 \mu \mathrm{g} \mathrm{ml}^{-1}$ bTMP for $20 \mathrm{~min}$ in the dark and then ultraviolet crosslinked at $360 \mathrm{~nm}$ for $10 \mathrm{~min}$. Genomic DNA was isolated using SDS and proteinase K digestion followed by phenol-chloroform extraction. Ultraviolet-dependent bTMP crosslinking of genomic DNA was confirmed by dot blot using HRP-conjugated streptavidin as a probe. For ChIP analysis, the sheared DNA was IPed with streptavidin Dynabeads M-280 (Invitrogen). The beads were washed as described above and the IPed DNA was eluted by boiling the beads in $95 \%$ formamide containing $10 \mathrm{mM}$ EDTA for $10 \mathrm{~min}$ at $90^{\circ} \mathrm{C}$. The eluted fractions were pooled and purified using the Qiagen PCR purification kit. The locus-specific enrichment was detected by $\mathrm{qPCR}$ normalized to the input DNA. A list of the primers used is shown in Supplementary Table 8.

Analysis of cell proliferation. The effect of SMARCA4 depletion on CH12F3-2A cell proliferation was analysed using CellTrace CFSE Cell Proliferation Kit (Molecular Probes) $^{69}$. Briefly, CH12F3-2A cells were transfected with siSMARCA4 or control siRNA (siCONT), and immediately labelled with $5 \mu \mathrm{M}$ of CFSE (carboxyfluorescein diacetate succinimidyl ester) for $15 \mathrm{~min}$ at $37^{\circ} \mathrm{C}$. CIT was added $24 \mathrm{~h}$ later and FACS analysis was performed at $24 \mathrm{~h}$ post stimulation on a Becton-Dickinson FACS-Calibur flow cytometer. The cells treated with Aphidicolin $\left(2 \mu \mathrm{g} \mathrm{ml}^{-1}\right)$, a well-known inhibitor of cell cycle progression, were used as a positive control.

Flow cytometry analysis of $\gamma \mathbf{H} 2 \mathrm{AX}$ phosphorylation. FACS-based $\mathrm{H} 2 \mathrm{AX}$ phosphorylation assay kit (Millipore, catalogue \# 17-344) was used to stain $\gamma \mathrm{H} 2 \mathrm{AX}$ in CH12F3-2A cells, essentially as described by the manufacturer. The cells were analysed on a Becton-Dickinson FACS-Calibur flow cytometer.

Construction of plasmids. The human SMARCA4 cDNA was PCR amplified using the pCMV5-BRG1-FLAG plasmid (Addgene\#17873) as a template, and cloned into the SalI/SmaI sites of the pEGFP-C2 vector. Constructs expressing mutant SMARCA4 (K785R or T910M) with defective ATPase activity were prepared by amplifying the WT construct using the indicated mutagenesis primers (Supplementary Table 8). Plasmid for the expression of GFP-SSRP1 was previously described $^{29}$. These constructs were used for CSR rescue experiments (see below) and reverse co-IPs in HEK 293T cells.

CSR complementation assay. To evaluate the requirement for ATPase activity of SMARCA4 in CSR, $2 \mu \mathrm{g}$ of the siRNA-resistant-WT or -mutant SMARCA4 expression plasmids (described above) were co-transfected along with SMARCA4 siRNA oligonucleotides into CH12F3-2A cells. The cells were cultured for $24 \mathrm{~h}$ and then treated with CIT for $24-48 \mathrm{~h}$. CSR complementation was analysed as described previously ${ }^{29}$.

\section{References}

1. Kerrigan, J. E. \& Pilch, D. S. A structural model for the ternary cleavable complex formed between human topoisomerase I, DNA, and camptothecin. Biochemistry 40, 9792-9798 (2001).

2. Pommier, Y. Topoisomerase I inhibitors: camptothecins and beyond. Nat. Rev. Cancer. 6, 789-802 (2006).

3. Tuduri, S. et al. Topoisomerase I suppresses genomic instability by preventing interference between replication and transcription. Nat. Cell Biol. 11, 1315-1324 (2009).

4. Kim, N. \& Jinks-Robertson, S. Transcription as a source of genome instability. Nat. Rev. Genet. 13, 204-214 (2012).

5. Miao, Z. H. et al. Nonclassic functions of human topoisomerase I: genome-wide and pharmacologic analyses. Cancer Res. 67, 8752-8761 (2007).

6. Aguilera, A. The connection between transcription and genomic instability. EMBO J. 21, 195-201 (2002).

7. Sordet, O., Nakamura, A. J., Redon, C. E. \& Pommier, Y. DNA double-strand breaks and ATM activation by transcription-blocking DNA lesions. Cell Cycle 9, 274-278 (2010).

8. Sordet, O. et al. Ataxia telangiectasia mutated activation by transcription- and topoisomerase I-induced DNA double-strand breaks. EMBO Rep. 10, 887-893 (2009).

9. Napierala, M., Bacolla, A. \& Wells, R. D. Increased negative superhelical density in vivo enhances the genetic instability of triplet repeat sequences. J. Biol. Chem. 280, 37366-37376 (2005).

10. Wojciechowska, M., Napierala, M., Larson, J. E. \& Wells, R. D. Non-B DNA conformations formed by long repeating tracts of myotonic dystrophy type 1 , myotonic dystrophy type 2, and Friedreich's ataxia genes, not the sequences per se, promote mutagenesis in flanking regions. J. Biol. Chem. 281, 24531-24543 (2006).

11. Hubert, Jr L., Lin, Y., Dion, V. \& Wilson, J. H. Topoisomerase 1 and single-strand break repair modulate transcription-induced CAG repeat contraction in human cells. Mol. Cell. Biol. 31, 3105-3112 (2011).

12. Takahashi, T., Burguiere-Slezak, G., Van der Kemp, P. A. \& Boiteux, S. Topoisomerase 1 provokes the formation of short deletions in repeated sequences upon high transcription in Saccharomyces cerevisiae. Proc. Natl Acad. Sci. USA 108, 692-697 (2011).

13. Lippert, M. J. et al. Role for topoisomerase 1 in transcription-associated mutagenesis in yeast. Proc. Natl Acad. Sci. USA 108, 698-703 (2011).

14. Kim, N. et al. Mutagenic processing of ribonucleotides in DNA by yeast topoisomerase I. Science 332, 1561-1564 (2011).

15. Alagoz, M., Chiang, S. C., Sharma, A. \& El-Khamisy, S. F. ATM deficiency results in accumulation of DNA-topoisomerase I covalent intermediates in neural cells. PLoS ONE 8, e58239 (2013).

16. Katyal, S. et al. Aberrant topoisomerase-1 DNA lesions are pathogenic in neurodegenerative genome instability syndromes. Nat. Neurosci. 17, 813-821 (2014).

17. Mosesso, P. et al. The novel human gene aprataxin is directly involved in DNA single-strand-break repair. Cell. Mol. Life Sci. 62, 485-491 (2005).

18. Shen, J. et al. Mutations in PNKP cause microcephaly, seizures and defects in DNA repair. Nat. Genet. 42, 245-249 (2010).

19. Muramatsu, M. et al. Class switch recombination and hypermutation require activation-induced cytidine deaminase (AID), a potential RNA editing enzyme. Cell 102, 553-563 (2000)

20. Nagaoka, H., Muramatsu, M., Yamamura, N., Kinoshita, K. \& Honjo, T. Activation-induced deaminase (AID)-directed hypermutation in the immunoglobulin Smu region: implication of AID involvement in a common step of class switch recombination and somatic hypermutation. J. Exp. Med. 195, 529-534 (2002).

21. Petersen, S. et al. AID is required to initiate Nbs1/gamma-H2AX focus formation and mutations at sites of class switching. Nature 414, 660-665 (2001).

22. Honjo, T. et al. The AID dilemma: infection, or cancer? Adv. Cancer Res. 113, 1-44 (2012).

23. Kobayashi, M. et al. AID-induced decrease in topoisomerase 1 induces DNA structural alteration and DNA cleavage for class switch recombination. Proc. Natl Acad. Sci. USA 106, 22375-22380 (2009).

24. Kobayashi, M. et al. Decrease in topoisomerase I is responsible for activationinduced cytidine deaminase (AID)-dependent somatic hypermutation. Proc. Natl Acad. Sci. USA 108, 19305-19310 (2011). 
25. Kato, L. et al. Nonimmunoglobulin target loci of activation-induced cytidine deaminase (AID) share unique features with immunoglobulin genes. Proc. Natl Acad. Sci. USA 109, 2479-2484 (2012).

26. Begum, N. A. \& Honjo, T. Evolutionary comparison of the mechanism of DNA cleavage with respect to immune diversity and genomic instability. Biochemistry 51, 5243-5256 (2012).

27. Blackwell, T. K. et al. Recombination between immunoglobulin variable region gene segments is enhanced by transcription. Nature 324, 585-589 (1986).

28. Bachl, J., Carlson, C., Gray-Schopfer, V., Dessing, M. \& Olsson, C. Increased transcription levels induce higher mutation rates in a hypermutating cell line. J. Immunol. 166, 5051-5057 (2001).

29. Stanlie, A., Aida, M., Muramatsu, M., Honjo, T. \& Begum, N. A. Histone3 lysine 4 trimethylation regulated by the facilitates chromatin transcription complex is critical for DNA cleavage in class switch recombination. Proc. Natl Acad. Sci. USA 107, 22190-22195 (2010).

30. Begum, N. A., Stanlie, A., Nakata, M., Akiyama, H. \& Honjo, T. The histone chaperone Spt6 is required for activation-induced cytidine deaminase target determination through H3K4me3 regulation. J. Biol. Chem. 287, 32415-32429 (2012).

31. Stanlie, A., Begum, N. A., Akiyama, H. \& Honjo, T. The DSIF subunits Spt4 and Spt5 have distinct roles at various phases of immunoglobulin class switch recombination. PLoS Genet. 8, e1002675 (2012).

32. Aida, M., Hamad, N., Stanlie, A., Begum, N. A. \& Honjo, T. Accumulation of the FACT complex, as well as histone H3.3, serves as a target marker for somatic hypermutation. Proc. Natl Acad. Sci. USA 110, 7784-7789 (2013).

33. Kato, L. et al. An evolutionary view of the mechanism for immune and genome diversity. J. Immunol. 188, 3559-3566 (2012).

34. Kadoch, C. et al. Proteomic and bioinformatic analysis of mammalian SWI/ SNF complexes identifies extensive roles in human malignancy. Nat. Genet. 45, 592-601 (2013)

35. Shain, A. H. \& Pollack, J. R. The spectrum of SWI/SNF mutations, ubiquitous in human cancers. PLoS ONE 8, e55119 (2013).

36. Clapier, C. R. \& Cairns, B. R. The biology of chromatin remodeling complexes. Annu. Rev. Biochem. 78, 273-304 (2009).

37. Wang, W. et al. Purification and biochemical heterogeneity of the mammalian SWI-SNF complex. EMBO J. 15, 5370-5382 (1996).

38. Franceschini, A. et al. STRING v9.1: protein-protein interaction networks, with increased coverage and integration. Nucleic Acids Res. 41, D808-D815 (2013).

39. Shannon, P. et al. Cytoscape: a software environment for integrated models of biomolecular interaction networks. Genome Res. 13, 2498-2504 (2003).

40. Smith, L. M., Willmore, E., Austin, C. A. \& Curtin, N. J. The novel poly(ADPRibose) polymerase inhibitor, AG14361, sensitizes cells to topoisomerase I poisons by increasing the persistence of DNA strand breaks. Clin. Cancer Res. 11, 8449-8457 (2005).

41. Okazaki, I. M. et al. Histone chaperone Spt6 is required for class switch recombination but not somatic hypermutation. Proc. Natl Acad. Sci. USA 108, $7920-7925$ (2011).

42. Okazaki, I. M., Kinoshita, K., Muramatsu, M., Yoshikawa, K. \& Honjo, T. The AID enzyme induces class switch recombination in fibroblasts. Nature 416, 340-345 (2002).

43. Faili, A. et al. AID-dependent somatic hypermutation occurs as a DNA single-strand event in the BL2 cell line. Nat. Immunol. 3, 815-821 (2002).

44. Nagaoka, H., Ito, S., Muramatsu, M., Nakata, M. \& Honjo, T. DNA cleavage in immunoglobulin somatic hypermutation depends on de novo protein synthesis but not on uracil DNA glycosylase. Proc. Natl Acad. Sci. USA 102, 2022-2027 (2005).

45. French, S. L. et al. Distinguishing the roles of Topoisomerases I and II in relief of transcription-induced torsional stress in yeast rRNA genes. Mol. Cell. Biol. 31, 482-494 (2011).

46. Chiarle, R. et al. Genome-wide translocation sequencing reveals mechanisms of chromosome breaks and rearrangements in B cells. Cell 147, 107-119 (2011).

47. Liu, M. et al. Two levels of protection for the B cell genome during somatic hypermutation. Nature 451, 841-845 (2008).

48. Yamane, A. et al. Deep-sequencing identification of the genomic targets of the cytidine deaminase AID and its cofactor RPA in B lymphocytes. Nat. Immunol. 12, 62-69 (2011).

49. Dykhuizen, E. C. et al. BAF complexes facilitate decatenation of DNA by topoisomerase IIalpha. Nature 497, 624-627 (2013).

50. Bartke, T. et al. Nucleosome-interacting proteins regulated by DNA and histone methylation. Cell 143, 470-484 (2010).

51. Safina, A. et al. Complex mutual regulation of facilitates chromatin transcription (FACT) subunits on both mRNA and protein levels in human cells. Cell Cycle 12, 2423-2434 (2013).

52. Shirakata, M. et al. HMG1-related DNA-binding protein isolated with V-(D)-J recombination signal probes. Mol. Cell. Biol. 11, 4528-4536 (1991).

53. Bruhn, S. L., Pil, P. M., Essigmann, J. M., Housman, D. E. \& Lippard, S. J. Isolation and characterization of human cDNA clones encoding a high mobility group box protein that recognizes structural distortions to DNA caused by binding of the anticancer agent cisplatin. Proc. Natl Acad. Sci. USA 89, 2307-2311 (1992).

54. Robbiani, D. F. \& Nussenzweig, M. C. Chromosome translocation, B cell lymphoma, and activation-induced cytidine deaminase. Annu. Rev. Pathol. 8, 79-103 (2013).

55. Kuppers, R. \& Dalla-Favera, R. Mechanisms of chromosomal translocations in B cell lymphomas. Oncogene 20, 5580-5594 (2001).

56. Leder, P. et al. Translocations among antibody genes in human cancer. Science 222, 765-771 (1983).

57. Wells, R. D. Non-B DNA conformations, mutagenesis and disease. Trends Biochem. Sci. 32, 271-278 (2007).

58. Denepoux, S. et al. Induction of somatic mutation in a human B cell line in vitro. Immunity 6, 35-46 (1997)

59. Smith, A. L., Friedman, D. B., Yu, H., Carnahan, R. H. \& Reynolds, A. B. ReCLIP (reversible cross-link immuno-precipitation): an efficient method for interrogation of labile protein complexes. PLoS ONE 6, el6206 (2011).

60. Petritis, B. O., Qian, W. J., Camp, 2nd D. G. \& Smith, R. D. A simple procedure for effective quenching of trypsin activity and prevention of 18O-labeling back-exchange. J. Proteome. Res. 8, 2157-2163 (2009).

61. Jorge, I. et al. Statistical model to analyze quantitative proteomics data obtained by $18 \mathrm{O} / 16 \mathrm{O}$ labeling and linear ion trap mass spectrometry: application to the study of vascular endothelial growth factor-induced angiogenesis in endothelial cells. Mol. Cell. Proteomics 8, 1130-1149 (2009).

62. Maita, N. et al. Human alpha-L-iduronidase uses its own N-glycan as a substrate-binding and catalytic module. Proc. Natl Acad. Sci. USA 110, 14628-14633 (2013).

63. Kikuchi, M. et al. Proteomic analysis of rat liver peroxisome: presence of peroxisome-specific isozyme of Lon protease. J. Biol. Chem. 279, 421-428 (2004).

64. Boboila, C. et al. Robust chromosomal DNA repair via alternative end-joining in the absence of X-ray repair cross-complementing protein 1 (XRCC1). Proc. Natl Acad. Sci. USA 109, 2473-2478 (2012).

65. Xu, J., Husain, A., Hu, W., Honjo, T. \& Kobayashi, M. APE1 is dispensable for S-region cleavage but required for its repair in class switch recombination. Proc. Natl Acad. Sci. USA 111, 17242-17247 (2014).

66. Schrader, C. E., Linehan, E. K., Mochegova, S. N., Woodland, R. T. \& Stavnezer, J. Inducible DNA breaks in Ig S regions are dependent on AID and UNG. J. Exp. Med. 202, 561-568 (2005).

67. Kiianitsa, K. \& Maizels, N. A rapid and sensitive assay for DNA-protein covalent complexes in living cells. Nucleic Acids Res. 41, e104 (2013).

68. Naughton, C. et al. Transcription forms and remodels supercoiling domains unfolding large-scale chromatin structures. Nat. Struct. Mol. Biol. 20, 387-395 (2013).

69. Stanlie, A., Yousif, A. S., Akiyama, H., Honjo, T. \& Begum, N. A. Chromatin reader Brd4 functions in Ig class switching as a repair complex adaptor of nonhomologous end-joining. Mol. Cell. 55, 97-110 (2014).

\section{Acknowledgements}

We thank all the members of the T.H. Laboratory for sharing reagents and technical information. This work was supported by the Ministry of Education, Culture, Sports, Science, and Technology of Japan Grant-in-Aid for Specially Promoted Research 17002015 (to T.H.), Grant-in-Aid for Scientific Research (C) 24590352 (to N.A.B.) and Grant-in-Aid for Scientific Research (C) 25440007 (to M.K.).

\section{Author contributions}

A.H., N.A.B., M.K. and T.H. designed the research; A.H., T.T. and N.A.B. performed the research; A.H., N.A.B., H.T., M.K. and T.H. analysed the data; and A.H., T.H. and N.A.B. wrote the manuscript.

\section{Additional information}

Supplementary Information accompanies this paper at http://www.nature.com/ naturecommunications

Competing financial interests: The authors declare no competing financial interests

Reprints and permission information is available online at http://npg.nature.com/ reprintsandpermissions/

How to cite this article: Husain, A. et al. Chromatin remodeller SMARCA4 recruits topoisomerase 1 and suppresses transcription-associated genomic instability. Nat. Commun. 7:10549 doi: 10.1038/ncomms10549 (2016).

This work is licensed under a Creative Commons Attribution 4.0 International License. The images or other third party material in this article are included in the article's Creative Commons license, unless indicated otherwise in the credit line; if the material is not included under the Creative Commons license, users will need to obtain permission from the license holder to reproduce the material. To view a copy of this license, visit http://creativecommons.org/licenses/by/4.0/ 